\title{
Black hole thermodynamics with dynamical lambda
}

\author{
Ruth Gregory, ${ }^{a, b}$ David Kastor $^{c}$ and Jennie Traschen ${ }^{c}$ \\ ${ }^{a}$ Centre for Particle Theory, Durham University, \\ South Road, Durham, DH1 3LE, U.K. \\ ${ }^{b}$ Perimeter Institute, 31 Caroline Street North, \\ Waterloo, ON, N2L 2Y5, Canada \\ ${ }^{c}$ Amherst Center for Fundamental Interactions, \\ Department of Physics, University of Massachusetts, \\ 710 N Pleasant St., Amherst, MA 01003, U.S.A. \\ E-mail: r.a.w.gregory@durham.ac.uk, kastor@umass.edu, \\ traschen@umass.edu
}

ABSTRACT: We study evolution and thermodynamics of a slow-roll transition between early and late time de Sitter phases, both in the homogeneous case and in the presence of a black hole, in a scalar field model with a generic potential having both a maximum and a positive minimum. Asymptotically future de Sitter spacetimes are characterized by ADM charges known as cosmological tensions. We show that the late time de Sitter phase has finite cosmological tension when the scalar field oscillation around its minimum is underdamped, while the cosmological tension in the overdamped case diverges. We compute the variation in the cosmological and black hole horizon areas between the early and late time phases, finding that the fractional change in horizon area is proportional to the corresponding fractional change in the effective cosmological constant. We show that the extended first law of thermodynamics, including variation in the effective cosmological constant, is satisfied between the initial and final states, and discuss the dynamical evolution of the black hole temperature.

KeYwords: Black Holes, Black Holes in String Theory

ARXIV EPRINT: 1707.06586 


\section{Contents}

1 Introduction 1

2 Pure de Sitter to de Sitter flows 3

2.1 Engineered flow 3

2.2 Slow-roll analysis 6

$\begin{array}{lll}2.3 & \text { Cosmic hair } & 7\end{array}$

3 Schwarzschild-quasi de Sitter spacetimes $\quad 11$

$\begin{array}{ll}3.1 \text { The scalar field behaviour } & 14\end{array}$

$\begin{array}{ll}3.2 \text { Growth of the event horizons } & 16\end{array}$

$\begin{array}{lll}3.2 .1 & \text { Cosmological event horizon } & 17\end{array}$

$\begin{array}{lll}3.2 .2 & \text { Black hole event horizon } & 17\end{array}$

4 Dynamical thermodynamics $\quad 19$

$\begin{array}{ll}4.1 \text { Analysis of horizon growth } & 19\end{array}$

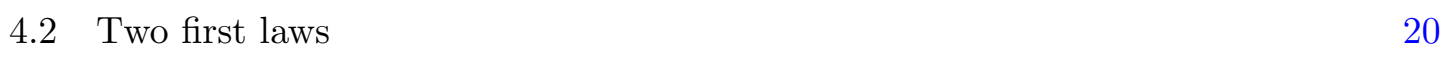

4.3 Temperature and mass for evolving black holes 20

$\begin{array}{llr}5 & \text { Illustrative example } & 22\end{array}$

6 Concluding remarks $\quad 25$

$\begin{array}{ll}\text { A Cosmological tension } & 25\end{array}$

\section{Introduction}

The physics of black holes in the early universe is an important subject, about which relatively little is known. The system is both interactive and dynamic, combining effects of cosmic expansion with accretion of matter onto the black hole. The gravitational thermodynamics is non-equilibrium, involving time dependent areas and surface gravities for the black hole and cosmological horizons, such that formulating an appropriate definition of temperature proves to be complicated. Still, physical implications of primordial black holes have been heavily researched, including a recent revival of interest that they may provide the dark matter and the progenitors of the massive black holes detected by LIGO [1-10]. In this paper we explore cosmological black holes in a relatively well-controlled setting that is also of physical interest, namely the evolution of black holes in slow-roll inflation. We consider transitions between distinct early and late time de Sitter phases, with evolution driven by a scalar field rolling slowly between a maximum and minimum of its potential, both of which are assumed to be positive. The initial and final states are described 
by Schwarzchild-de Sitter (SdS) metrics with different values of the black hole mass and cosmological constant.

We begin in section 2 by analyzing pure vacuum to vacuum transitions, without a black hole present. Exact results are obtained for an 'engineered' example and compared with the results of a perturbative slow-roll calculation. The growth of the cosmological horizon between the two de Sitter vacua is found to obey the extended first law [11, 12], which in the absence of a black hole is given by

$$
T \delta S=\mathcal{V} \delta P
$$

where the pressure $P$ is provided by the effective cosmological constant, $\mathcal{V}$ is the thermodynamic volume, and $T$ is the initial temperature of the de Sitter horizon. Hence $\delta P$ is dynamically generated by the scalar field. Depending on the parameters of the scalar field potential there are two qualitatively distinct ways that the metric can approach the late time de Sitter metric. The motion of the scalar field is either overdamped or underdamped as it settles into the true vacuum, corresponding to a slow-roll or an oscillatory relaxation respectively. Although in both cases the metric decays exponentially fast to de Sitter, the ADM cosmological tension [13] is infinite for the overdamped case, but finite for the oscillatory evolution. This behavior is analogous to behavior found for AdS black holes with scalar fields, as well as in studies of AdS domain-wall/cosmology dualities [14-29] in which a scalar field gets a negative mass-squared from resting at the maximum of a potential, and back-reaction generates an infinite ADM mass.

In the second part of the paper, we add a black hole to the cosmology and solve for the evolution of the scalar field and metric in a perturbative "slow-roll" approximation. This generalizes the calculations of [30] to potentials in which both the initial and final states are approximately de Sitter, and we focus on thermodynamic aspects of the evolution. The black hole grows due to accretion of the scalar field, but the cosmological horizon is subject to competing influences. While the growing black hole tends to pull the cosmological horizon further in, the decaying cosmological constant makes it expand. We find that the expansion dominates. For a potential interpolating between initial and final values of the cosmological constant, $\Lambda_{i}$ and $\Lambda_{f}$, we find that the change in both the black hole and cosmological horizon areas evolve proportionally to $|\delta \Lambda|=\left|\Lambda_{f}-\Lambda_{i}\right|$ times a factor that depends on properties of the Schwarzchild-de Sitter (SdS) spacetime. This is summarized in equation (4.1) below. We then show that the extended first law of thermodynamics [11, 12], that relates the sum of $T \delta S$ contributions from each horizon to $\mathcal{V} \delta P$, is satisfied between the initial and final SdS phases.

The area growth calculations only depend on the temperatures of the background static spacetime, but one would like to go further and work with a dynamical temperature. As a first step, we utilize a definition of the dynamical black hole temperature $T_{d y n}$ based on the Kodama vector [31]. This is very nearly that of a Schwarzschild-de Sitter black hole with the instantaneous values of the effective cosmological constant and black hole radius. We show how the black hole temperature relaxes from its initial value to its final asymptotic value, at a rate dependent on the rolling of the scalar and the local horizon 
surface gravity. In section 5, we demonstrate this explicitly for a simple potential, finding analytic expressions for the dynamical area and temperature.

\section{Pure de Sitter to de Sitter flows}

Our goal in this paper is to investigate the effect of a dynamically generated cosmological constant on the growth of black hole and cosmological horizons, and to explore the thermodynamic relations for such evolving black hole systems. We begin by examining a pseudo-de Sitter spacetime, where the cosmological constant varies in time, with no blackhole present. As noted in [25], this is a double-analytically continued version of an AdS flow, thus an analogue of the C-theorem [32-34] tells us that the cosmological constant must always flow to lower values in time. In accordance with this, we consider a real scalar field $\phi$ with potential $W(\phi)$ and assume that $W(\phi)$ has a maximum $W_{i}$ at $\phi=\phi_{i}$, and a minimum, $W_{f}$ at $\phi=\phi_{f}$. If the scalar field starts off at $\phi_{i}$ at early times, and rolls to $\phi_{f}$ at late times, the cosmological constant $\Lambda$ will make a transition between the values $W_{i} / M_{p}^{2}$ to $W_{f} / M_{p}^{2}$, where $M_{p}^{2}=1 / 8 \pi G$, at early and late times.

We take the action for the coupled Einstein plus scalar field system to be

$$
S=\frac{1}{2} \int d^{4} x \sqrt{-g}\left(-M_{p}^{2} R+(\nabla \phi)^{2}-2 W(\phi)\right)
$$

and use a mostly minus signature. Assuming an FRW form for the metric $d s^{2}=d \tau^{2}-$ $a^{2}(\tau) d \mathbf{x}^{2}$, the equations of motion for the system are given by

$$
\begin{aligned}
\left(\frac{\dot{a}}{a}\right)^{2} & =\frac{1}{3 M_{p}^{2}}\left[\frac{1}{2} \dot{\phi}^{2}+W(\phi)\right] \\
\ddot{\phi}+3 H \dot{\phi}+\frac{\partial W}{\partial \phi} & =0
\end{aligned}
$$

where $H=\dot{a} / a$. For the pure dS-dS flow, we can simply numerically integrate these FRW equations for any desired potential, however for the analysis of the black hole set-up, it is useful to have analytic solutions, or approximate solutions, to use to explore the dynamical evolution of the horizons.

\subsection{Engineered flow}

As a first method, we start with an interpolating Ansatz for the scalar field, and use the Hamilton-Jacobi formulation [35] to engineer a potential $W(\phi)$ that corresponds to this flow. This approach has been used, for example, to generate smooth analytic domain wall solutions in the presence of gravity [36], and is very similar to the "fake supersymmetry" approach described in [37].

Briefly, the Hamilton-Jacobi approach uses $\phi$ as a time coordinate, writing $H=H(\phi)$. The equations of motion (2.2) then imply that

$$
H^{\prime}=-\frac{\dot{\phi}}{2 M_{p}^{2}}
$$


where the superscript prime denotes a derivative with respect to $\phi$. With this identification, the Friedmann equation takes the form of a first order NLDE for $H(\phi)$

$$
H^{\prime 2}-\frac{3 H^{2}}{2 M_{p}^{2}}+\frac{W(\phi)}{2 M_{p}^{4}}=0
$$

A judicious choice of evolution for $\phi(\tau)$ that allows $\dot{\phi}$ to be re-expressed as a function of $\phi$ then gives an $H^{\prime}$ that can be integrated up to give $H$ and hence $W(\phi)$ using (2.4).

For example, if we suppose that the flow of the scalar is

$$
\phi(\tau)=\eta \tanh (\sqrt{\lambda} \eta \tau)
$$

then we find

$$
H^{\prime}=-\frac{\sqrt{\lambda}}{2 M_{p}^{2}}\left(\eta^{2}-\phi^{2}\right) \quad \Rightarrow \quad H(\phi)=H_{0}-\frac{\sqrt{\lambda}}{6 M_{p}^{2}} \phi\left(3 \eta^{2}-\phi^{2}\right)
$$

where $H_{0}$ is an integration constant. The Hamilton-Jacobi equation (2.4) then finally determines the scalar field potential to be

$$
W(\phi)=3 M_{p}^{2}\left(H_{0}-\frac{\sqrt{\lambda}}{6 M_{p}^{2}} \phi\left(3 \eta^{2}-\phi^{2}\right)\right)^{2}-\frac{\lambda}{2}\left(\phi^{2}-\eta^{2}\right)^{2}
$$

Taking $H_{0}>0$, the potential $W(\phi)$ has a local maximum at $\phi=-\eta$ and a minimum at $\phi=\eta$. Correspondingly, the Hubble parameter makes a transition from a larger value $H_{i}$ associated with the higher vacuum energy at early times, to a smaller value $H_{f}$ for the lower vacuum energy at late times, given by

$$
H_{i}=H_{0}+\sqrt{\lambda} \eta^{3} / 3 M_{p}^{2}, \quad H_{f}=H_{0}-\sqrt{\lambda} \eta^{3} / 3 M_{p}^{2},
$$

Integrating the expression for $H(\tau)$, obtained by plugging (2.5) into (2.6), yields the scale factor

$$
\log a(\tau)=H_{0} \tau-\frac{\eta^{2}}{3 M_{p}^{2}} \log \cosh (\sqrt{\lambda} \eta \tau)+\frac{\eta^{2}}{12 M_{p}^{2}} \operatorname{sech}^{2}(\sqrt{\lambda} \eta \tau)
$$

We see that for $|\tau| \gtrsim 1 / \sqrt{\lambda} \eta, \ln a$ is approximately linear in $\tau$ with the appropriate Hubble constants given by (2.8), thus $\ln a$ is a smoothed out step function, as we would expect.

In the late time limit, the cosmological scale factor and scalar field are approximately given by

$$
a(\tau) \simeq K e^{H_{f} \tau}\left(1-\frac{\eta^{2}}{2 M_{p}^{2}} e^{-H_{f} \Gamma \tau}\right) \quad, \quad \phi \simeq \eta\left(1-2 e^{-H_{f} \Gamma \tau / 2}\right)
$$

where $K=2^{\left(\eta^{2} / 3 M_{p}^{2}\right)}$ and we have defined $\Gamma H_{f}=4 \sqrt{\lambda} \eta$ in order to facilitate comparison to the late time behavior in subsequent examples. Note that with this notation, the expression for the scalar field (2.5) becomes $\phi(\tau)=\eta \tanh \left(H_{f} \Gamma \tau / 4\right)$. For fixed $H_{f}$ the parameter $\Gamma$ then allows one to interpolate between slow-roll behavior for $\Gamma \ll 1$ and a sudden change for $\Gamma \gg 1$. 
We are interested in the evolution of the future cosmological horizon. If a light signal is emitted at time $\tau$, then as the reception time goes to infinity the signal is received at a co-moving coordinate separation $r_{c}(\tau)$ away

$$
r_{c}(\tau)=\int_{\tau}^{\infty} \frac{d \tau^{\prime}}{a\left(\tau^{\prime}\right)}
$$

The cosmological horizon radius $d_{c}(\tau)$ is the corresponding proper distance

$$
d_{c}(\tau)=a(\tau) r_{c}(\tau)
$$

Evaluating the integral (2.11) for the cosmological horizon radius $d_{c}(\tau)$ numerically with the scale factor (2.9) gives a smooth evolution between the initial Hubble horizon, $H_{i}^{-1}$, and the final horizon, $H_{f}^{-1}$, depicted in figure 1 by a blue line. It is interesting to compare this exact result to the approximate solution for $r_{c}$ which is gotten by using a step-function approximation for $a(\tau)$,

$$
a(\tau) \approx e^{H_{i} \tau} \Theta(-\tau)+e^{H_{f} \tau} \Theta(\tau)
$$

Performing the integral (2.11) in this case gives an approximate expression for the evolution of the cosmological horizon radius

$$
d_{c}(\tau) \approx\left[\frac{1}{H_{i}}+\left(\frac{1}{H_{f}}-\frac{1}{H_{i}}\right) e^{H_{i} \tau}\right] \Theta(-\tau)+\frac{1}{H_{f}} \Theta(\tau)
$$

figure 1 shows the approximate expression compared to the exact one. We see in both the exact and approximate results that the cosmological horizon interpolates between $d_{c}=$ $1 / H_{i}$ at early times and $d_{c}=1 / H_{f}$ at late times. These correspond respectively to the Killing horizons of the early and late time de Sitter phases, for the Killing vectors $\xi_{*}=$ $(\partial / \partial \tau)-H_{*} \sum_{j} x^{j}\left(\partial / \partial x^{j}\right)$, where $H_{*}=H_{i}$ or $H_{f}$ respectively. It is satisfying to see that even the simple step-function approximation for $a(\tau)$ captures the teleological behavior of the horizon as $\tau$ increases to the transition time $\tau=0$.

It is interesting to look at the change in the cosmological horizon area $A_{c}=4 \pi d_{c}^{2}$ over the evolution from early to late times, which is given by

$$
\delta A_{c}=4 \pi\left(\frac{1}{H_{f}^{2}}-\frac{1}{H_{i}^{2}}\right)
$$

An extended first law for de Sitter black holes, including variation in the cosmological constant was derived in [11, 12]. For the case of no black hole (see also [38]), this reduces to

$$
T_{c} \delta S_{c}=\mathcal{V} \delta P
$$

where $T_{c}$ is the temperature of cosmological horizon, $S_{c}=A_{c} / 4$ is its entropy, $P=-\Lambda / 8 \pi$ is the cosmological pressure, and $\mathcal{V}$ is called the thermodynamic volume. The extended first law, in this simple case, relates the change in cosmological horizon entropy to the change in cosmological constant. For a de Sitter spacetime the thermodynamic volume is equal to

$$
\mathcal{V}=\frac{4 \pi d_{c}^{3}}{3}
$$




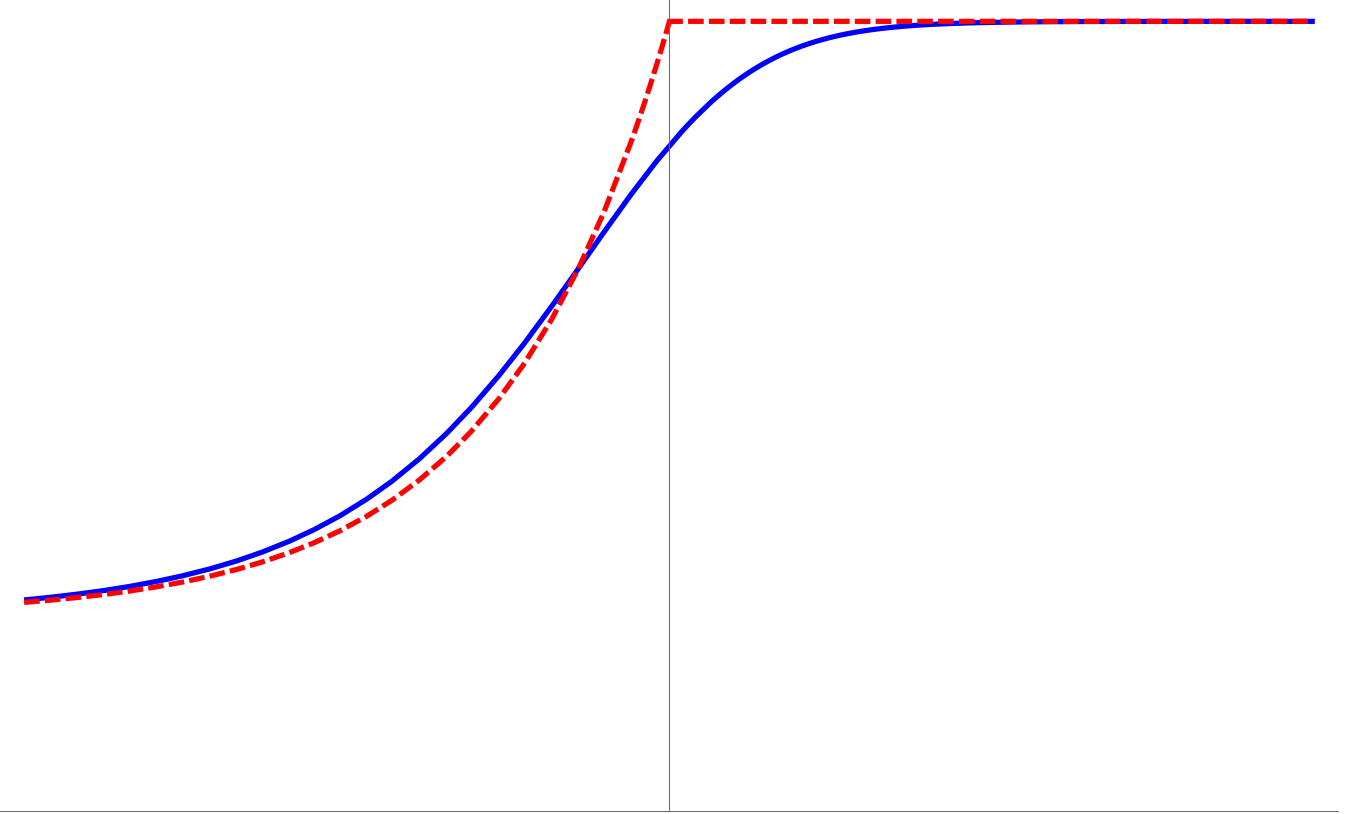

Figure 1. The actual cosmological horizon evolution for the Hamilton-Jacobi engineered potential (blue) vs. the approximation (red-dashed).

i.e. the volume of a Euclidean sphere of radius $d_{c}$, while the horizon temperature is $T=1 /\left(2 \pi d_{c}\right)$. If we consider a limiting case of our evolution such that $H_{f}=H_{i}+\delta H$, where $\delta H / H_{i} \ll 1$, then the change in horizon area (2.15) is given to leading order by

$$
\delta A_{c}=-\frac{8 \pi \delta H}{H_{i}^{3}}
$$

and it is easily verified that the first law (2.16) is satisfied. In section 4 we will see that the first law for a slow-roll inflationary spacetime with a black hole also obeys the appropriate extension of (2.16).

\section{$2.2 \quad$ Slow-roll analysis}

In the previous subsection we assumed a form for $\phi$ that interpolated between two de Sitter phases, and found the exact potential and scale factor, such that the cosmological evolution was a prescribed flow from the maximum of the potential to the minimum. The approach to the true vacuum was overdamped rather than oscillatory, corresponding to a slow-roll evolution. In this section we show that if one starts with a classic double well potential, with positive minima, then the behavior of the scalar field is qualitatively the same as that previously assumed.

For an analytic treatment we take the standard slow roll assumption [39] that the scalar field evolution is friction dominated, so that

$$
3 H \dot{\phi} \simeq-W^{\prime}(\phi)
$$


Now define slow-roll parameters

$$
\varepsilon(\phi)=\frac{M_{p}^{2}}{2} \frac{W^{\prime 2}}{W^{2}} \ll 1, \quad \Gamma(\phi)=2 M_{p}^{2} \frac{W^{\prime \prime}}{W} \ll 1
$$

The second parameter $\Gamma$ (related to the standard slow roll "eta" parameter by a factor of two) is relevant for evolution near a minimum of $W$, where $\varepsilon=0$. While $\phi$ is rolling slowly, it will be approximately linear in cosmological time, although the behaviour near each critical point will be modified.

Now let $W$ be a double well potential with a $\max$ at $\phi=0$ and a min at $\phi=\eta$,

$$
W(\phi)=W_{f}\left(1+\frac{\Gamma}{16 \eta^{2} M_{p}^{2}}\left(\phi^{2}-\eta^{2}\right)^{2}\right)
$$

where $\Gamma=\Gamma(\eta)$ is the slow-roll parameter evaluated at the minimum. The Einstein constraint equation evaluated at late times, when $\phi=\eta$, gives the standard relation between the effective cosmological constant $\Lambda_{f}=W_{f} / M_{p}^{2}$ and the Hubble parameter in the final de Sitter phase,

$$
H_{f}^{2}=\frac{W_{f}}{3 M_{p}^{2}}
$$

The parameter $\varepsilon \sim \eta^{2} \Gamma^{2} / M_{p}^{2}$, and we assume that $\Gamma, \varepsilon \ll 1$. Since the time dependent part of $\phi$ is already first order, in its equation of motion (2.19) we can approximate $H \approx H_{f}$. The solution for $\phi$ is then

$$
\phi^{2}=\frac{\eta^{2}}{2} \operatorname{sech}\left(\frac{H_{f} \Gamma}{4} \tau\right) e^{H_{f} \Gamma \tau / 4}=\frac{\eta^{2}}{2}\left[1+\tanh \left(\frac{H_{f} \Gamma}{4} \tau\right)\right]
$$

Here an integration constant determining the transition point has been set to zero, but one has the freedom to replace $\tau$ in $(2.23)$ with $\left(\tau-\tau_{0}\right)$. Hence we see that for the double well potential (2.21) the scalar field has a $\tanh \left(H_{f} \Gamma \tau\right)$ type behavior, similar to the engineered case. This makes sense since the potentials (2.7) and (2.21) have the same qualitative features for evolving from the maximum to a minimum. At late times the field goes like

$$
\phi \simeq \eta\left(1-\frac{1}{2} e^{-H_{f} \Gamma \tau / 2}\right)
$$

which has the same decay rate to the true vacuum as in the previous example (2.10). The scale factor approaches its final de Sitter form with corrections that fall off like $e^{-H_{f} \Gamma \tau}$.

\subsection{Cosmic hair}

As shown in [13], asymptotically future de Sitter (AFdS) spacetimes carry cosmic hair, encoded in the exponential fall-off terms in the metric, analogous to the ADM charges of a black hole. For black holes, the ADM integrals are computed at spatial infinity, while for AFdS spacetimes, these integrals are computed at future infinity. With the AFdS boundary conditions established in [13], the exponentially small size of the corrections to de Sitter in this regime are compensated by the exponentially growing spatial volume, giving 
finite results for what were referred to as cosmological tension charges. ${ }^{1}$ While e.g. the ADM mass and angular momentum of a black hole are associated with asymptotic time translation and rotation symmetries, cosmological tension is associated with asymptotic spatial translation symmetries. It is analogous to the ADM spatial tension charges defined for black brane spacetimes in [41] (see also [42] for the asymptotically AdS case). It was found [13] that cosmological tension in a given spatial direction captures the leading order correction of the scale factor in that direction to its limiting late time de Sitter behavior.

In this paper, we consider transitions between early and late time de Sitter phases. Although it diverts us briefly from our main line of development, we explore in this section how cosmological tension behaves in the Einstein-scalar field cosmologies considered here. These represent an interesting and qualitatively different set of examples from those explored in [13], where we can compute the cosmological tensions associated with the approach to the late time de Sitter limit. Assuming that the minimum of the potential is at a finite value of $\phi$, we find that the cosmological tensions will be finite, if the approach to the minimum is underdamped. However, in the overdamped case, the corrections to de Sitter behavior fall off too slowly, and the cosmological tension charges diverge.

In both the engineered example in section 2.1 and the approximate analytic solution for the slow-roll example in section 2.2, the scalar field and metric indeed decay to the late time de Sitter vacuum exponentially fast. This agrees with expectations based on [40], which showed that an initially expanding, homogeneous spacetime with cosmological constant $\Lambda>0$, matter fields satisfying the weak $(\rho \geq 0)$ and strong $(\rho+3 p \geq 0)$ energy conditions, and non-positive spatial curvature, falls off to de Sitter exponentially quickly at late times. To align our scalar field system with the assumptions of [40], we take the cosmological constant to be given in terms of the final value of the scalar potential $\Lambda=W_{f} / M_{p}^{2}$. The scalar field then moves in the shifted potential

$$
U(\phi)=W(\phi)-W_{f} \geq 0
$$

For the scalar field evolution the energy density and pressure are given by

$$
\rho=\frac{1}{2} \dot{\phi}^{2}+U(\phi) \geq 0, \quad p=\frac{1}{2} \dot{\phi}^{2}-U(\phi) \geq 0
$$

Since $\rho \geq 0$ the weak energy condition is satisfied. However, $\rho+3 p=2\left(\dot{\phi}^{2}-U(\phi)\right)$, which is negative in the early time de Sitter phase, so that the strong energy condition is not satisfied. Nonetheless, we find that the metric and scalar field still approach the late time de Sitter vacuum exponentially fast. This decay is generic, determined by the fact that near a minimum of the potential the wave equation for $\phi$ becomes that of a damped simple harmonic oscillator, with damping provided by the Hubble expansion. However, we show that in the overdamped case the detailed decay rate is too slow to result in a finite cosmological tension charge.

Assume that the scalar field potential $W(\phi)$ has a maximum and a minimum so that there can be two de Sitter phases as the field evolves from an initial value $\phi_{i}$ at the

\footnotetext{
${ }^{1}$ Cosmic hair in the form of cosmological tension charges is fully consistent with the results of reference [40], even though this work is often mischaracterized as a "cosmic no-hair theorem".
} 
maximum $W_{i}$ to a final value $\phi_{f}$ at the minimum $W_{f}$, as in the two examples above. We want to solve equations (2.2) for $\phi(\tau)$ and $a(\tau)$ at late times as $\phi$ approaches the minimum at $\phi_{f}$. Assume that the second derivative of the potential is non-zero at $\phi_{f}$, so that near the minimum the scalar field potential can be approximated by

$$
W(\phi) \simeq W_{f}+\frac{1}{2} W_{f}^{\prime \prime}\left(\phi-\phi_{f}\right)^{2}
$$

To leading order the wave equation (2.2) for $\phi$ then reduces to the ODE for a damped simple harmonic oscillator

$$
\ddot{\phi}+3 H_{f} \dot{\phi}+W_{f}^{\prime \prime}\left(\phi-\phi_{f}\right)=0
$$

which has the solutions

$$
\phi(\tau)=\phi_{f}+\phi_{1} e^{-\beta_{ \pm} H_{f} \tau}, \quad \beta_{ \pm}=\frac{3}{2}(1 \pm \sqrt{1-2 \Gamma / 3})
$$

where $\phi_{1}$ is a constant and $\Gamma=2 W_{f}^{\prime \prime} / 3 H_{f}^{2}$. Overdamped oscillation results from real values of $\beta$, corresponding to $\Gamma \leq 3 / 2$, and in this case $\beta_{-}$is the dominant mode. From (2.20) we see that slow-roll evolution corresponds to $\Gamma \ll 1$, giving

$$
\phi=\phi_{f}+\phi_{1} e^{-\Gamma H_{f} \tau / 2} \quad \text { (slow-roll) }
$$

in agreement with the late time slow-roll evolution in (2.24).

The late time behavior of $\phi$ determines the late time behavior of the scale factor. As $\phi$ approaches its value at the minimum of the potential $W(\phi)$ the Einstein constraint equation becomes

$$
\left(\frac{\dot{a}}{a}\right)^{2} \simeq \frac{1}{3 M_{p}^{2}}\left(W_{f}+\frac{1}{2} W_{f}^{\prime \prime}\left(\phi-\phi_{f}\right)^{2}+\frac{1}{2} \dot{\phi}^{2}\right)
$$

the evolution of the scale factor in this regime can be written as

$$
a(\tau) \simeq e^{H_{f} \tau}+\delta a(\tau)
$$

where the deviation $\delta a(\tau)$ from the late time de Sitter evolution is small. The leading terms relate the late time value of the Hubble parameter to the value of the potential at its minimum, as in (2.22), while the next order terms give

$$
\delta a(\tau)=-\frac{\phi_{1}^{2}}{24 M_{p}^{2} \beta_{-}}\left(\beta_{-}^{2} H_{f}^{2}+W_{f}^{\prime \prime}\right) e^{H_{f}\left(1-2 \beta_{-}\right) \tau}
$$

In the slow-roll approximation, with $\Gamma \ll 1$, the late time limit of the scale factor is then

$$
a(\tau)=e^{H_{f} \tau}\left(1-\frac{\phi_{1}^{2} H_{f}^{2}}{8 M_{p}^{2}} e^{-\Gamma H_{f} \tau}\right) \quad \text { (slow-roll) }
$$

in agreement with (2.10). Critically damped motion occurs when $\Gamma=3 / 2$. In this case the two roots coincide, and the second linearly independent mode goes like $e^{-\beta H_{f} \tau} \ln \tau$. 
Underdamped oscillations occur when $\Gamma>3 / 2$ [35], and at this point there is a qualitative change in the aymptotic behavior of $\phi$ and $a(\tau)$ as the fields oscillate around the minimum with decaying amplitude,

$$
\begin{aligned}
\phi(\tau) & =\phi_{f}+\phi_{1} e^{-3 H_{f} \tau / 2} \sin \omega \tau \\
a & =e^{H_{f} \tau}\left(1-e^{-3 H_{f} \tau}\left[\frac{1}{36 M_{p}^{2}} \phi_{1}^{2} W_{f}^{\prime \prime}+C_{1} \cos 2 \omega \tau+C_{2} \sin 2 \omega \tau\right]\right)
\end{aligned}
$$

where $\omega^{2}=W_{f}^{\prime \prime}-H_{f}^{2}$, and $C_{1}$ and $C_{2}$ are constants whose precise expressions will not be needed. The underdamped, ${ }^{2}$ oscillatory, cases all decay at the same rate, which is precisely that needed for a finite, non-zero, cosmological tension ${ }^{3} \mathcal{T}$. The ADM charges are defined in terms of boundary integrals, which require a number of further definitions in order to specify. We present this material in a short appendix to the paper. In computing $\mathcal{T}$ we average over a period, and the oscillatory terms average to zero. For the non-oscillatory solutions (2.33), the key ingredient in the boundary integral is the behavior of a term like

$$
\frac{\delta \dot{a}}{a_{d s}} d v \simeq e^{H_{f}\left(3-2 \beta_{-}\right) \tau}
$$

where $d v=e^{3 H_{f} \tau}$ is the area element on a constant time slice of the background de Sitter metric. As $\tau \rightarrow \infty$ this is finite and non-zero only for the critically damped case of $\beta_{-}=3 / 2$, whereas the integral diverges for all the overdamped cases which have $\beta_{-}<3 / 2$. However, in the critically damped case the two modes corresponding to $\beta_{ \pm}$coincide, and the dominant late time mode is instead a second linearly independent solution that goes like $(\ln \tau) e^{-3 H_{f} \tau / 2}$ and also leads to a divergent tension. Hence it is only the oscillatory modes that yield a finite cosmological tension $\mathcal{T}$. Since the tension involves an integration over an infinite spatial area, we make the spatial coordinates periodic with period $L$, and the finite quantity is the tension per unit area. For the underdamped case, where the tension is finite, one then finds

$$
\frac{2 \mathcal{T}}{M_{p}^{2} L^{2}}=\frac{9}{4} \phi_{1}^{2} H_{f}
$$

where $\phi_{1}^{2}$ sets the magnitude of $\phi-\phi_{f}$ and $\delta a$.

To summarize, there are two qualitatively distinct ways for $\phi$ to decay to the minimum of the potential, either with or without oscillations. These behaviors are distinguished by finite or infinite cosmological tension. Note that if $\phi$ were coupled to other fields to reheat the universe, this would also distinguish different physical mechanisms for the reheating.

Lastly, it is interesting to see how these different asymptotics look in AdS. In four dimensions the metric of a planar AdS black hole approaches AdS at spatial infinity exponentially fast, like $e^{-3 y / \ell}$, where $y$ is a proper length radial coordinate and $\ell$ is the AdS length scale. However, the relevant area element grows like $e^{3 y / \ell}$, so the resulting boundary term for the ADM mass is finite. A significant amount of research has been done on the behavior

\footnotetext{
${ }^{2}$ Note that the Hamilton-Jacobi method in section 2.1 will only produce monotonic scalar flows and hence will not generate oscillatory cases such as those considered here.

${ }^{3}$ Since the metric is isotropic, all three tensions, associated with invariance in the three spatial directions, are the same.
} 
of scalar fields in AdS/ CFT, and particularly analogous to the issue of future asymptotically de Sitter spacetime are studies of AdS black holes with scalar hair, AdS domain-wall scenarios, and holographic domain walls [14-29]. In these situations the potential for the scalar field is negative, and the field approaches a maximum of the potential, which provides an effective negative mass-squared. The two modes of the field decay like $e^{-\Delta_{ \pm} y / \ell}$,

$$
\Delta_{ \pm}=\frac{3}{2}\left(1 \pm \sqrt{1+4 m^{2} \ell^{2} / 9}\right), \quad m^{2}=W_{\infty}^{\prime \prime}<0
$$

with the constraint $m_{B F}^{2} \leq m^{2} \leq m_{B F}^{2}+1<0$, where $m_{B F}^{2}=-9 /\left(4 \ell^{2}\right)$ is the Breitenlohner-Freedman bound [14, 16-21, 43]. This formula is analogous to the scalar field decay in the cosmological case (2.29), with the substitution of a negative potential in which $\phi$ approaches a maximum, for the positive potential with $\phi$ approaching a minimum. The dominant far field mode with $\Delta_{-}$decays too slowly for the spacetime to have a finite ADM mass, as we have found for the late time de Sitter behavior with real $\beta_{-}$. In the AdS case a finite ADM charge can be constructed by combining contributions from the gravitational and scalar fields $[15,16,22,23]$. A similar situation arises in AdS domain wall spacetimes in which a scalar field potential interpolates in the radial direction between two AdS vacua. The interpolation between AdS vacua corresponds to an RG flow between two CFTs, and has previously been compared to de Sitter to de Sitter transitions [24]. A topic for future analysis is to work out the combination of cosmological tension with a scalar field contribution to form a finite generalized ADM tension, appropriate for a slow roll approach to de Sitter.

\section{Schwarzschild-quasi de Sitter spacetimes}

In section 2 we analyzed de Sitter to de Sitter evolutions, with no black hole present. We now investigate the effects of a black hole on this transition, and in particular how the black hole and cosmological horizons evolve. Exact solutions of dynamical black holes include the McVittie metric [44], which is simply SdS in cosmological coordinates in the most physical case, and examples of maximally charged multi-cosmological black holes both without [45] and with scalar fields [46]. Accretion of fields and growth of cosmological black holes has been studied in different approximations and numerically, addressing scalar field cosmologies, generalizing the properties of Killing black holes, and the dynamics of accretion [31, 38, 47-62].

In this paper we follow the approach of [30] and systematically apply perturbation theory and the slow roll approximation to the Einstein-scalar field equations, identify the horizons, and use well-behaved coordinates on the horizons to compute the behavior of the scalar field and thermodynamic properties. Our results yield analytic expressions for the evolution of horizon areas, temperatures, thermodynamic volume, and local pressure. We find that the generalised first law of thermodynamics is satisfied between the final and initial SdS states. Though the results are given in terms of a general potential $W(\phi)$, it is most straightforward to make a thermodynamic interpretation in the case that $W$ has a maximum and a minimum, since then the initial and final states are equilibria 
described by a static SdS metric. The main results of this section are summarized in equations (3.37), (3.40), and (3.42), (3.45), which give the total change in the cosmological and black hole horizon areas respectively. The reader uninterested in the derivation can skip to those results without loss of continuity.

For a slowly rolling scalar field, the spacetime will be adiabatically de Sitter (or SdS), with small, "time-dependent" corrections. The idea therefore is to first find a solution with a constant $\phi$, then to correct this perturbatively for a rolling $\phi$. Given that we have a cosmological evolution in 'time', together with the black hole giving us a 'radial' dependence of our geometry, our analysis should capture the dependence on these two coordinates. Since the black hole and cosmological event horizons represent coordinate singularities in the standard SdS metric, we follow the methodology of reference [30] that treated black hole evolution for a scalar with an exponential potential, using null coordinates for the metric that encode the dependence on two parameters:

$$
d s^{2}=4 e^{2 \nu} \sqrt{\frac{B_{0}}{B}} d U d V-B d \Omega_{\mathrm{II}}^{2}
$$

but are nonsingular at the horizons, and indeed facilitate the analysis of horizon behaviour that are now located at " $U=0$ " or " $V=0$ ". Here, $B_{0}^{1 / 2}$ is a fiducial length scale that maintains dimensional consistency, and $d \Omega_{\mathrm{II}}^{2}$ is the metric on the 2-sphere. This form of the metric also clearly identifies the main physical degree of freedom of the gravitational field as the $B$-function, since $\nu$ communicates with the remaining gauge freedom of conformal transformations in the $U, V$ plane. In the absence of any scalar evolution, the physical degree of freedom in $B$ corresponds to the mass of the black hole as we now briefly review. (This discussion follows [30,63]).

In this null gauge, the Einstein equations become

$$
\begin{aligned}
\phi_{, U V} & =-W_{, \phi}(\phi) \sqrt{\frac{B_{0}}{B}} e^{2 \nu}-\frac{1}{2 B}\left(B_{, U} \phi_{, V}+B_{, V} \phi_{, U}\right) \\
B_{, U V} & =2\left(\frac{W(\phi)}{M_{p}^{2}} B^{1 / 2}-B^{-1 / 2}\right) e^{2 \nu} B_{0}^{1 / 2} \\
\nu_{, U V} & =\frac{1}{2}\left(\frac{W(\phi)}{M_{p}^{2}} B^{-1 / 2}+B^{-3 / 2}\right) e^{2 \nu} B_{0}^{1 / 2}-\frac{\phi_{, U} \phi_{, V}}{2 M_{p}^{2}} \\
B_{, V V} & =2 \nu_{, V} B_{, V}-B \phi_{, V}^{2} / M_{p}^{2} \\
B_{, U U} & =2 \nu_{, U} B_{, U}-B \phi_{, U}^{2} / M_{p}^{2}
\end{aligned}
$$

If $\phi$ is constant, equations (3.5) and (3.6) give

$$
2 \nu=\log \frac{B_{, V}}{\sqrt{B_{0}}}+G^{\prime}(U)=\log \frac{B_{, U}}{\sqrt{B_{0}}}+F^{\prime}(V)
$$

where $F$ and $G$ are in principle arbitrary functions, expressed here as derivatives for convenience. From this, we deduce that

$$
B=B[F(V)+G(U)] \text { and } e^{2 \nu} B_{0}^{1 / 2}=F^{\prime} G^{\prime} B^{\prime}
$$


Then (3.3) can be integrated up to give

$$
B^{\prime}=\frac{4 W_{0}}{3 M_{p}^{2}} B^{3 / 2}-4 B^{1 / 2}+\mu
$$

where the integration constant $\mu$ is nonzero if a black hole is present. Substituting these expressions back into the metric gives

$$
d s^{2}=-16\left(1-\frac{W_{0}}{3 M_{p}^{2}} B-\frac{\mu}{4 \sqrt{B}}\right) d F d G-B d \Omega_{\mathrm{II}}^{2} .
$$

Comparison with the SdS metric suggests that we identify $\mu=8 G M$, and choose a radial coordinate $r=\sqrt{B}$. It is then fairly clear that if we identify $F$ and $G$ with the advanced and retarded null coordinates

$$
F \leftrightarrow-\frac{\left(t+r^{\star}\right)}{4}, \quad G \leftrightarrow \frac{\left(t-r^{\star}\right)}{4}
$$

(where $r^{\star}$ is the standard tortoise coordinate), then transform to $t, r$ coordinates we recover the SdS metric:

$$
d s^{2}=N(r) d t^{2}-\frac{d r^{2}}{N(r)}-r^{2} d \Omega_{I I}^{2} .
$$

We have written the SdS potential as

$$
N(r)=1-\frac{2 G M}{r}-H_{0}^{2} r^{2}=-\frac{H_{0}^{2}}{r}\left(r-r_{c}\right)\left(r-r_{h}\right)\left(r-r_{N}\right)
$$

where $H_{0}^{2}=W_{0} / 3 M_{p}^{2}$, and we identify the black hole horizon (if present) as $r_{h}$, the cosmological horizon as $r_{c}$, and the remaining zero of $N$ as $r_{N}=-\left(r_{c}+r_{h}\right)$. Note, the roots of $N$ are related to the physical parameters via

$$
\begin{aligned}
\Lambda & =3 H^{2}=3 /\left(r_{c}^{2}+r_{h}^{2}+r_{h} r_{c}\right) \\
2 G M & =H^{2} r_{c} r_{h}\left(r_{c}+r_{h}\right)
\end{aligned}
$$

and the tortoise coordinate $r^{\star}$ is given explicitly by

$$
r^{\star}(r)=\int \frac{d r}{N(r)}=\frac{1}{2 \kappa_{c}} \log \left|\frac{r-r_{c}}{r_{c}}\right|+\frac{1}{2 \kappa_{h}} \log \left|\frac{r-r_{h}}{r_{h}}\right|+\frac{1}{2 \kappa_{N}} \log \left|\frac{r-r_{N}}{r_{N}}\right| .
$$

Here, $\kappa_{i}$ are the usual surface gravities at the individual horizons, $2 \kappa_{i}=N^{\prime}\left(r_{i}\right)$.

Finally, although $F$ and $G$ are null coordinates, the metric still has coordinate singularities at the black hole and cosmological event horizons. These can of course be removed locally by using the standard Kruskal coordinates:

$$
\begin{array}{lll}
\text { - } r \rightarrow r_{c} & V=\frac{1}{2 \kappa_{c}} \exp \left[\kappa_{c}\left(t+r^{\star}\right)\right], & U=\frac{1}{2 \kappa_{c}} \exp \left[\kappa_{c}\left(t-r^{\star}\right)\right] \\
\text { - } r \rightarrow r_{h} & v=\frac{1}{2 \kappa_{h}} \exp \left[\kappa_{h}\left(t+r^{\star}\right)\right], & u=-\frac{1}{2 \kappa_{h}} \exp \left[-\kappa_{h}\left(t-r^{\star}\right)\right]
\end{array}
$$

however, no global maximal extension of the SdS coordinates is possible. Given that we are interested in the future evolution of the spacetime, we could choose to use $\{u, V\}$ as nonsingular coordinates, however, in practice it is easier to analyse physics near the event horizons in the local Kruskals. 


\subsection{The scalar field behaviour}

Prior to starting the analysis of an evolving black hole, it is useful to relate this notation to our previous discussion with cosmological time. With the SdS black hole, the natural solution is expressed in static gauge, as we have discussed above, but it is useful to see how the null and static coordinates relate to the cosmological coordinates.

Setting $\mu=0$, and integrating (3.9) gives

$$
B[X]=\frac{1}{H_{0}^{2}} \tanh ^{2}\left[-2 H_{0} X\right]
$$

where $X=F+G<0$. Using (3.11), (3.15), and $\kappa_{c}=-H_{0}=-r_{c}^{-1}$,

$$
X=-\frac{r^{\star}}{2}=\frac{1}{4 H_{0}} \ln \left|\frac{1-H_{0} r}{1+H_{0} r}\right|
$$

Taking $V \pm U$ and using (3.16) then relates these expressions to our canonical cosmological coordinates (via conformal time $\hat{\eta}=\int d t / a$ ) as

$$
\begin{aligned}
\tau & =-\frac{1}{H_{0}} \ln \left[-H_{0}(U+V)\right]=t+\frac{1}{2 H_{0}} \log \left(1-H_{0}^{2} r^{2}\right) \\
\rho & =(V-U)=\frac{r e^{-H_{0} t}}{\sqrt{1-H_{0}^{2} r^{2}}}
\end{aligned}
$$

In the cosmological slow-roll approximation with no black hole, $\phi$ only depends on cosmological time $\tau$. Even though this is a more involved expression in the static gauge, we still have the notion that $\phi$ depends on a single function of $t$ and $r$. In [30], it was found that in the slow-roll approximation for the case of an exponential scalar potential, in the presence of a black hole $\phi$ depended linearly on a variable $x(t, r)$, which was a generalization of the cosmological time coordinate in (3.19). Here we maintain this notion of slow-roll, and look for a similar $x(t, r)$ that reduces to $\tau$ as the black hole area goes to zero.

In this slow-roll approximation, derivatives of $\phi$ and $W^{\prime}(\phi)$ are assumed to be small quantities relative to the overall magnitude of the potential, thus (3.3)-(3.6) reduce to the pure cosmological constant equations we have just discussed, and (3.2) to leading order requires only these background forms of the metric functions to find the evolution of $\phi$ due to its potential in the presence of the black hole. Substituting in these forms, (3.2) is

$$
\frac{\phi_{, F G}}{N(r)}-\frac{2}{r}\left(\phi_{, F}+\phi_{, G}\right)=4 \frac{\partial W}{\partial \phi}
$$

(recalling that $r$ is a function of $F+G$ ).

We now look for a variable $x(t, r)$, such that $\phi$ is predominantly a function of $x$. That is, $x$ must be suitably chosen to render (3.20) an ODE for $\phi$ when the $\phi^{\prime \prime}$ term is neglected. Now

$$
\frac{\phi_{, F G}}{N(r)}-\frac{2}{r}\left(\phi_{, F}+\phi_{, G}\right)=\frac{x_{, F} x_{, G}}{N} \phi^{\prime \prime}+\left[\frac{x_{, F G}}{N}-2 \frac{x_{, F}+x_{, G}}{r}\right] \phi^{\prime}
$$

so let

$$
x=t+\xi[r]
$$


then,

$$
\begin{aligned}
& x_{, F}=2+\xi^{\prime} \frac{B^{\prime}}{2 \sqrt{B}}=2\left(1-N \xi^{\prime}\right) \\
& x_{, G}=-2+\xi^{\prime} \frac{B^{\prime}}{2 \sqrt{B}}=-2\left(1+N \xi^{\prime}\right)
\end{aligned}
$$

and (3.20) becomes

$$
\frac{N^{2} \xi^{\prime 2}-1}{N} \phi^{\prime \prime}+\frac{\left(r^{2} N \xi^{\prime}\right)^{\prime}}{r^{2}} \phi^{\prime}=\frac{\partial W}{\partial \phi}
$$

We can now read off our requirement for slow-roll as

$$
\xi^{\prime} \propto \frac{C+r^{3}}{r^{2} N}
$$

The final determination of the constant of proportionality and the integration constant $C$ is determined by the boundary conditions that the field is purely ingoing at the black hole horizon and purely outgoing at the cosmological horizon, leading to

$$
x=t-r^{\star}+\frac{1}{\kappa_{h}} \ln \left|\frac{r-r_{h}}{r_{h}}\right|+\frac{r_{c}}{2 \kappa_{h} r_{h}} \ln \left|\frac{r-r_{N}}{r_{N}}\right|-\frac{r_{h} r_{c}}{r_{c}-r_{h}} \ln \frac{r}{r_{0}}
$$

where $r^{\star}$ is given in (3.15). The new $x$ coordinate reduces to the expression for the cosmological coordinate $\tau$ in (3.19) when $r_{h}=0$.

This implies that

$$
\xi^{\prime}=\frac{1}{N}\left(\frac{r_{c}^{2} r_{h}^{2}\left(r_{c}+r_{h}\right)-r^{3}\left(r_{c}^{2}+r_{h}^{2}\right)}{r^{2}\left(r_{c}^{3}-r_{h}^{3}\right)}\right)
$$

and dropping the $\phi^{\prime \prime}$ term, the slow-roll equation becomes

$$
3 \gamma \phi^{\prime}(x)=-\frac{\partial W}{\partial \phi}
$$

where

$$
\gamma=\frac{r_{c}^{2}+r_{h}^{2}}{r_{c}^{3}-r_{h}^{3}}=\frac{A_{\mathrm{tot}}}{3 \mathcal{V}}
$$

Here $A_{\text {tot }}=4 \pi\left(r_{c}^{2}+r_{h}^{2}\right)$ is the total horizon area, and $\mathcal{V}=4 \pi\left(r_{c}^{3}-r_{h}^{3}\right) / 3$ the thermodynamic volume, of the de Sitter black hole system. The volume $\mathcal{V}$, with $r_{h}=0$, arose in the thermodynamics of the cosmological horizon in the absence of a black hole, equations (2.16) and (2.17). It will again enter the thermodynamics of the black hole-cosmological system in subsequent sections.

It is interesting to compare (3.28) to the cosmological slow-roll equation, where $\gamma=H_{0}$. Clearly as $r_{h} \rightarrow 0$, the two slow-roll equations coincide, but we can now explicitly see the effect of the black hole on the friction for $\phi$-motion. As $r_{h}$ is switched on, the denominator in (3.29) decreases and the numerator increases, hence $\gamma$ is larger for larger black holes at fixed $\Lambda .{ }^{4}$ Thus the effect of a black hole is to further slow down the slow roll inflation,

\footnotetext{
${ }^{4}$ It is a little more subtle, since for fixed $H^{2}$, i.e. $W, r_{c}$ decreases as $r_{h}$ increases, however, it is easy to check that the overall effect of increasing the black hole size is to increase $\gamma$.
} 
and for black holes very close to the Nariai limit, the evolution becomes arbitrarily slow. Indeed, expanding $\gamma$ for small and large black holes, demonstrates this effect clearly:

$$
\gamma \sim \begin{cases}H_{0}\left(1+G M H_{0}\right) & M \rightarrow 0 \\ \frac{2}{3\left(r_{c}-r_{h}\right)} & r_{h} \rightarrow r_{c}\end{cases}
$$

Finally, given that the coefficient of the $\phi^{\prime \prime}$ term contains a $1 / N$ factor, we must check that this term remains small. From (3.27), we see that $N \xi^{\prime} \rightarrow \pm 1$, at the black hole and cosmological horizons, thus the term multiplying $\phi^{\prime \prime}$ is in fact regular at the horizons, and thus overall stays small. It is therefore consistent to drop the second derivative term as long as $\left|\phi^{\prime \prime}\right| \ll \gamma \phi^{\prime}$.

\subsection{Growth of the event horizons}

What does the resulting evolution look like? Two fundamental geometrical properties of the spacetime are the areas of the black hole and cosmological horizons. Since the black hole is accreting the scalar field, we expect the black hole to grow. A bigger black hole will tend to pull in the cosmological horizon. However, the effective cosmological constant is decreasing, which leads the cosmological horizon to grow. We will see that the second effect dominates, and that both horizons grow.

Ideally, we would calculate the full gravitational back reaction throughout the spacetime, as described in [30], however, this process is rather involved, and somewhat specious to the main theme of our discussion, namely the evolution of the event horizons. As it turns out, this is fairly straightforward to extract. Note that in the background solution, the horizon (a null surface) is at fixed $r$, i.e. fixed $B$. Taking the local Kruskals at each horizon, given in equation (3.16), the cosmological event horizon is defined as $V=0$, and parametrised by $U$ whereas the black hole event horizon is defined as $u=0$, and parametrised by $v$. In the vicinity of the horizons of the background $\operatorname{SdS}$ we have:

$$
\begin{cases}V=U \exp \left[2 \kappa_{c} r^{\star}\right] \approx U\left(r-r_{c}\right) & \text { as } r \rightarrow r_{c} \\ u=-\frac{1}{4 \kappa_{h}^{2} v} \exp \left[2 \kappa_{h} r^{\star}\right] \approx-\frac{1}{4 \kappa_{h}^{2} v}\left(r-r_{h}\right) & \text { as } r \rightarrow r_{h}\end{cases}
$$

To get the horizon growth, the idea is to expand the Einstein equations (3.5) for the black hole horizon, and (3.6) for the cosmological horizon, by using the fact that $B_{, u}\left(B_{, V}\right)$ is zero on the black hole (cosmological) event horizon in the background spacetime.

$$
\begin{array}{rlr}
\text { - } r \rightarrow r_{c} & \delta B_{, U U}=2 \nu_{, U} \delta B_{, U}-B \phi_{, U}^{2} / M_{p}^{2} \\
\text { - } r \rightarrow r_{h} & \delta B_{, v v}=2 \nu_{, v} \delta B_{, v}-B \phi_{, v}^{2} / M_{p}^{2}
\end{array}
$$

Here the derivative of $\phi$ terms enter as a perturbative source. Lastly, we will need the expression for $\nu$ at each horizon:

$$
\begin{aligned}
& \text { - } r \rightarrow r_{c} \quad e^{2 \nu}=\frac{r N(r)}{4 \kappa_{c}^{2} U V} \approx-\frac{r_{c} N}{4 \kappa_{c}^{2} U^{2}\left(r-r_{c}\right)} \approx-\frac{r_{c}}{2 \kappa_{c} U^{2}} \\
& \text { - } r \rightarrow r_{h} \quad e^{2 \nu}=-\frac{r N(r)}{4 \kappa_{h}^{2} u v} \approx \frac{r_{h} N}{\left(r-r_{h}\right)} \approx 2 \kappa_{h} r_{h}
\end{aligned}
$$




\subsubsection{Cosmological event horizon}

Starting with the cosmological event horizon, as $r \rightarrow r_{c}, x \sim \kappa_{c}^{-1} \ln \left(2 \kappa_{c} U\right)+$ const., and from (3.34) we have $\nu_{, U} \simeq-1 / U$ along the cosmological horizon. Hence (3.32) gives

$$
\begin{aligned}
(U \delta B)_{, U U} & =-\frac{r_{c}^{2}}{\kappa_{c}^{2} M_{p}^{2}} \frac{\phi^{\prime 2}}{U}=-\frac{r_{c}^{2}}{\kappa_{c} M_{p}^{2}} \phi^{\prime 2} \frac{d x}{d U} \\
\Rightarrow \quad(U \delta B)_{, U} & =-\frac{r_{c}^{2}}{\kappa_{c} M_{p}^{2}} \int \phi^{\prime 2} d x=\frac{r_{c}^{2}}{3 \gamma \kappa_{c} M_{p}^{2}} \int \frac{\partial W}{\partial \phi} d \phi \\
& =-\frac{r_{c}^{2}}{3 \gamma \kappa_{c} M_{p}^{2}}\left(W_{i}-W[\phi(U)]\right)
\end{aligned}
$$

Here we have set $\delta B=0$ when $\phi$ takes its initial value $\phi_{i}$, which is as $U \rightarrow-\infty$ and $W_{i}=W\left(\phi_{i}\right)$. Integrating again gives

$$
\delta B=-\frac{r_{c}^{2}}{3 \gamma\left|\kappa_{c}\right| M_{p}^{2} U} \int_{U}^{0}\left(W_{i}-W\left[\phi\left(U^{\prime}\right)\right]\right) d U^{\prime}
$$

where we have written $-\kappa_{c}=\left|\kappa_{c}\right|$ to clarify that the change in the horizon area is positive. As $U \rightarrow-\infty, \phi \rightarrow \phi_{i}$, and we get $\delta B \rightarrow 0$, but as we go to future infinity, or $U \rightarrow 0$, then $\phi \rightarrow \phi_{f}$ and

$$
\delta B=\frac{1}{3 \gamma\left|\kappa_{c}\right| M_{p}^{2}} r_{c}^{2}\left(W_{i}-W_{f}\right),
$$

This means that the total change in cosmological horizon radius is positive, and given by

$$
\delta r_{c}=\delta \sqrt{B\left(r_{c}\right)}=\frac{\delta B}{2 r_{c}}=\frac{1}{6 \gamma\left|\kappa_{c}\right| M_{p}^{2}} r_{c}\left(W_{i}-W_{f}\right)
$$

When there is no black hole, then $r_{h}=0, r_{c}=1 / H,\left|\kappa_{c}\right|=H$, and the change in horizon radius is $H_{f}^{-1}-H_{i}^{-1}$, in agreement with equation (2.14) and figure 1.

Finally, this gives for the change in the cosmological horizon area $\delta A_{c}=8 \pi r_{c} \delta r_{c}$

$$
\delta A_{c}=\frac{A_{c}}{3 \gamma\left|\kappa_{c}\right| M_{p}^{2}}\left(W_{i}-W_{f}\right)
$$

This can be written in terms of the change in the early and late time effective cosmological constants by using $\Lambda_{I}=W_{I} / M_{p}^{2}$. Since $W_{i}>W_{f}$, corresponding to evolution of $\phi$ by rolling down the potential, the cosmological horizon grows.

It is of interest to find how much the presence of a black hole affects the growth of the cosmological horizon, for a fixed potential. In equation (3.40) the quantities $A_{c}, \kappa_{c}$ and $\gamma$ all depend on $r_{h}$. Looking at the limits, the effect is of course negligible for very small black holes, but as the size of the black hole horizon becomes comparable to that of the cosmological horizon, the total change in $A_{c}$ is diminished by a factor of approximately two-thirds.

\subsubsection{Black hole event horizon}

The analysis for the black hole horizon area is similar to that for cosmological horizon, but now we use the Kruskal coordinates $u, v$. The black hole horizon is at $u=0$, and 
$v$ is the coordinate along the horizon. As $r \rightarrow r_{h}$, we have $x \sim \kappa_{h}^{-1} \ln \left(2 \kappa_{h} v\right)+$ const. From equation (3.34) it follows that $\nu$ is constant on the event horizon, and the perturbed equation for $B$ becomes

$$
\delta B_{, v v}=-\frac{r_{h}^{2}}{\kappa_{h}^{2} M_{p}^{2} v^{2}} \phi^{2}
$$

which is solved by

$$
\delta B=-\frac{r_{h}^{2}}{3 \gamma \kappa_{h} M_{p}^{2}} v \int_{v}^{\infty}\left(W\left[\phi\left(v^{\prime}\right)\right]-W[\phi(0)]\right) \frac{d v^{\prime}}{v^{\prime 2}}
$$

We have set a constant of integration equal to zero that would have lead to an increase in the area that grows linearly in $v$, rather than a constant value as is consistent with asymptotically dS boundary conditions. As $v \rightarrow 0$, we get $\delta B \rightarrow 0$, but as $v \rightarrow \infty$ (or as we go to future infinity),

$$
\delta B=\frac{r_{h}^{2}}{3 \gamma \kappa_{h} M_{p}^{2}}\left(W_{i}-W_{f}\right)
$$

This means that the change in black hole horizon radius is

$$
\delta r_{h}=\delta \sqrt{B\left(r_{h}\right)}=\frac{\delta B}{2 r_{h}}=\frac{r_{h}}{6 \gamma \kappa_{h} M_{p}^{2}}\left(W_{i}-W_{f}\right)
$$

One sees that $\delta B_{, v} \neq 0$ at $v=0$, which is equivalent to the observation that the event horizon begins to move out before matter has crossed it, which follows from the teleological nature of its definition. The corresponding change in the area of the black hole horizon is

$$
\delta A_{h}=\frac{A_{h}}{3 \gamma \kappa_{h} M_{p}^{2}}\left(W_{i}-W_{f}\right)
$$

The area of the black hole horizon increases, as is expected due to accretion of the scalar field. The fractional rate of area increase is less for the black hole than for the cosmological horizon, since (3.40) and (3.45) imply

$$
\frac{\left(\delta A_{h} / A_{h}\right)}{\left(\delta A_{c} / A_{c}\right)}=\frac{\left|\kappa_{c}\right|}{\kappa_{h}}<1
$$

Now that we have the changes in horizon radii, as a check we can compute the change in $\Lambda$ that would be required by the SdS relation equation (3.14), using the changes in horizon radius computed by integrating the horizon radii, equations (3.39) and (3.44)

$$
\begin{aligned}
\delta \Lambda & =-3 H^{4}\left(\left(2 r_{c}+r_{h}\right) \delta r_{c}+\left(2 r_{h}+r_{c}\right) \delta r_{h}\right) \\
& =\frac{H^{4}\left(W_{f}-W_{i}\right)}{\gamma M_{p}^{2}}\left(\left(2 r_{c}+r_{h}\right) \frac{r_{c}}{2 \kappa_{c}}-\left(2 r_{h}+r_{c}\right) \frac{r_{h}}{2 \kappa_{h}}\right) \\
& =\frac{H^{4} \delta W}{\gamma M_{p}^{2}}\left(\frac{r_{c}^{2}}{H^{2}\left(r_{c}-r_{h}\right)}-\frac{r_{h}^{2}}{H^{2}\left(r_{c}-r_{h}\right)}\right)=\frac{\delta W}{M_{p}^{2}}
\end{aligned}
$$

as required. 


\section{Dynamical thermodynamics}

In this section we explore a number of aspects of the thermodynamics of the slow roll evolution of black holes between initial and final Schwarzschild-de Sitter states that we have established in the last section.

\subsection{Analysis of horizon growth}

Let us further analyze the results for evolution of the black hole and cosmological horizons found in the last section. Using the definition (3.29) of $\gamma$ as well as the thermodynamic volume and total horizon area, both equations (3.40) and (3.45) can be written in terms of thermodynamic quantities as

$$
\delta A_{\alpha}=\frac{\mathcal{V}}{2 \pi T_{\alpha}} \frac{A_{\alpha}}{A_{\text {tot }}}\left(\Lambda_{i}-\Lambda_{f}\right), \quad \alpha=h, c
$$

where $2 \pi T_{\alpha}=\left|\kappa_{\alpha}\right|$ are the horizon temperatures and $\Lambda_{i, f}=W_{i, f} / M_{p}^{2}$ are the initial and final values of the cosmological constant. For a fixed potential $W$, and hence fixed values for $\Lambda_{i, f}$, how much does a black hole with initial radius $r_{h}$ grow? Using the formulae for the temperatures and areas in Schwarzschild-de Sitter spacetime (3.14), we can express the change in the areas in terms of $r_{h}, \Lambda_{i}$, and $\delta \Lambda=\Lambda_{i}-\Lambda_{f}$. For the black hole horizon, one finds that

$$
\delta A_{h}=A_{h} \frac{|\delta \Lambda|}{\Lambda_{i}} \frac{2 r_{h}\left(r_{c}^{2}+r_{h}^{2}+r_{c} r_{h}\right)}{\left(2 r_{h}+r_{c}\right)\left(r_{h}^{2}+r_{c}^{2}\right)}
$$

while the corresponding expression for $\delta A_{c}$ is obtained by interchanging $r_{h}$ and $r_{c}$. However, this is not quite what we want, since $r_{c}$ is still dependent on $r_{h}$ and $\Lambda_{i}$. This dependence can be dealt with exactly using (3.14), but it is most useful to focus on the limits where the black hole horizon is either small or comparable in size to the cosmological horizon. One finds that in these limits, the change in the black hole horizon area is given by

$$
\begin{aligned}
\delta A_{h} & \simeq 2 A_{h} \frac{|\delta \Lambda|}{\sqrt{3} \Lambda_{i}} \times\left(r_{h} \sqrt{\Lambda_{i}}\right), & & r_{h} \sqrt{\Lambda_{i}} \ll 1 \\
& \simeq A_{h} \frac{|\delta \Lambda|}{\Lambda_{i}}, & & r_{h} \sqrt{\Lambda_{i}} \sim 1
\end{aligned}
$$

We see that the fractional growth in area, $\delta A_{h} / A_{h}$, is parametrically suppressed for small black holes, while it is of order $|\delta \Lambda| / \Lambda_{i}$ for large ones. Likewise, one can ask how much the cosmological horizon is "pulled back" by the black hole, compared to the case with no black hole. In the limiting cases of small and large black holes, one finds that the change in the cosmological horizon area is given by

$$
\begin{aligned}
\delta A_{c} & \simeq 12 \pi \frac{|\delta \Lambda|}{\Lambda_{i}^{2}}, \quad r_{h} \sqrt{\Lambda_{i}} \ll 1 \\
& \simeq 4 \pi \frac{|\delta \Lambda|}{\Lambda_{i}^{2}}, \quad r_{h} \sqrt{\Lambda_{i}} \sim 1
\end{aligned}
$$

For small black holes, the effect of the black hole on the cosmological horizon growth is negligible. While for large black holes, it can be reduced by as much as $2 / 3$. For a black hole with initial area $1 / 100$ of the cosmological horizon area, one finds that the diminution effect is a factor of $1 / 10$. 


\subsection{Two first laws}

Two independent first laws for asymptotically de Sitter black hole spacetimes can be derived [12]. One relates the change in area of the black hole horizon to the change in mass, while the other relates the change in area of the cosmological horizon to the change in mass. Including the possibility of a change in the cosmological constant, each of these laws has an additional term proportional to a thermodynamic volume times $\delta \Lambda$, i.e. a term of the form $\mathcal{V}_{\alpha} \delta \Lambda$, where $\alpha=h, c$. One can take the difference of the two first laws, such that the mass term drops out giving

$$
T_{h} \delta S_{h}+T_{c} \delta S_{c}=\mathcal{V} \delta P
$$

where $\mathcal{V}$ is the thermodynamic volume between the black hole and cosmological horizons, which was introduced above, and we have set $\left|\kappa_{I}\right| \delta A_{I}=8 \pi T_{I} \delta S_{I}$ and $\Lambda=-8 \pi P$.

Here we are studying the evolution from one Schwarzschild-de Sitter spacetime to another, where the change is effected by the rolling scalar field. Combining the changes in the horizon areas computed in the previous sections, and given in equations (3.40), and (3.45), one can check that (4.5) is indeed satisfied for these evolutions. This requires use of (3.29), which implies that $\left(A_{h}+A_{c}\right) /(3 \gamma)=\mathcal{V}$. This result is interesting because a dynamical scalar field is beyond the scope of applicability of the derivation in [12], and yet our results for a black hole in slow-roll inflation are still found to satisfy the first law, applied to the differences between the initial and final de Sitter phases. This agreement suggests that the first law might be satisfied continuously along the evolution, an idea that we return to in the discussion.

\subsection{Temperature and mass for evolving black holes}

A definition of temperature for dynamical black holes was discussed in [31], which proposes that a generalized surface gravity is

$$
2 \kappa_{d y n}=-\star d \star d \sqrt{B}
$$

where the Hodge dual $\star$ refers to the $2 \mathrm{D}$ spacetime perpendicular to $\theta$ and $\phi$, or the $U-V$ part, and the right hand side is evaluated on the horizon. ${ }^{5}$ On the $u-v$ subspace, one has

$$
\star d u=d u, \quad \star d v=-d v, \quad \star d u \wedge d v=-\left(g_{u v}^{-1}\right)
$$

Evaluating (4.6) on the black hole horizon at $u=0$, one finds that

$$
\begin{aligned}
2 \kappa_{d y n}=-\star d \star d \sqrt{B} & =-\star d\left[\frac{B_{, u} d u-B_{, v} d v}{2 \sqrt{B}}\right]=\left[-\frac{B_{, u v}}{2}+\frac{B_{, u} B_{, v}}{4 B}\right] \frac{e^{-2 \nu}}{\sqrt{B_{0}}} \\
& =B^{-1 / 2}-\frac{W(\phi)}{M_{p}^{2}} \sqrt{B}+\frac{B_{, u} B_{, v}}{4 B} e^{-2 \nu} B_{0}^{-1 / 2}
\end{aligned}
$$

where the Einstein equation (3.6) has been used in obtaining the second line. One might guess that for slow-roll evolution the black hole temperature would instantaneously be

\footnotetext{
${ }^{5}$ This formula differs by a minus sign (4.6) from that in [31] due to using different signatures.
} 
that of a Schwarzschild-de Sitter spacetime with the value of $\Lambda$ and $r_{h}$ at that time, and this turns out to be almost the case. Using the function $N(r)$ in (3.13), the black hole temperature in Schwarzschild-de Sitter is $4 \pi T_{s d s}=\frac{1}{r_{h}}-\Lambda r_{h}$. For the evolving spacetime, this gives the temperatures in the initial and final states, where $r_{h}$ and $\Lambda$ taking their initial and final values. Defining a quasi-static temperature that interpolates between the initial and final values along a sequence of Schwarzschild-de Sitter spacetimes as

$$
4 \pi T_{q s}(v)=\frac{1}{r_{h}(v)}-\frac{W(v)}{M_{p}^{2}} r_{h}(v)
$$

then this matches the first two terms in (4.8). We can evaluate the last term in (4.8) perturbatively. In the background solution, one has $B_{, v}=0$ and $B_{, u} e^{-2 \nu} B_{0}^{-1 / 2}=-4 \kappa_{h} v$, so that

$$
\frac{B_{, u} B, v}{4 B} e^{-2 \nu} B_{0}^{-1 / 2} \simeq-\frac{\kappa_{h} v}{r_{h}^{2}} \delta B_{, v}
$$

So the dynamical temperature is given by

$$
2 \pi T_{d y n}(v)=2 \pi T_{q s}(v)-\frac{\kappa_{h} v}{r_{h}^{2}} \delta B_{, v}
$$

where the derivative of $\delta B$, which follows from (3.41) (or from (3.42)), is given by

$$
\delta B_{, v}=-\frac{r_{h}^{2}}{3 \gamma \kappa_{h} M_{p}^{2}}\left[\int_{v}^{\infty} \delta W\left[\phi\left(v^{\prime}\right)\right] \frac{d v^{\prime}}{v^{\prime 2}}-\frac{\delta W[\phi(v)]}{v}\right]
$$

Now consider the late time behaviour of this temperature, when the integral in $\delta B$ is approximately given by $^{6} \int \delta W / v^{2} \simeq \delta W / v$. Substituting this in to $\delta B$ and (4.12), at late times the dynamical temperature is given by the quasi-static approximation, which expanded to first order is

$$
T_{d y n} \simeq T_{q s} \simeq T_{s d s, i}-\frac{\left(W_{i}-W[\phi(v)]\right)}{24 \pi \gamma \kappa_{h} M_{p}^{2}}\left(1+\frac{W_{i} r_{h}^{2}}{M_{p}^{2}}-6 \gamma \kappa_{h} r_{h}^{2}\right)
$$

where $T_{s d s, i}$ is the temperature of the initial SdS spacetime.

A definition of the dynamical mass can be found by considering the first law relating the change in mass to the changes in black hole area and $\Lambda$. Perturbations about a static black hole with positive $\Lambda$ satisfy [12]

$$
\delta M=T_{h} \delta S_{h}-\mathcal{V}_{h} \delta P
$$

where $\mathcal{V}_{h}=4 \pi r_{h}^{3} / 3$ is the black hole thermodynamic volume, here given for SdS spacetime. As noted in the context of the first law formulated between the two horizons (4.5), a dynamical cosmological constant due to a scalar field potential is beyond the scope of the derivation of (4.14). However, since we found that (4.5) is true for our solutions, let us assume that (4.14) also holds as well and see what it implies for the mass. This is equivalent

\footnotetext{
${ }^{6}$ For more detailed discussion of the asymptotic behaviour of $B$, see section 5 , where the dynamics of the black hole system is computed in detail for a sample potential.
} 
to assuming that at late times, when the stress-energy is again dominated by the smaller effective $\Lambda_{f}$, that the metric can be put into static SdS form with the evolved values of $r_{h}$ and $r_{c}$. The final mass $M_{f}$ is then given by the SdS relations (3.14). Explicitly, substituting $\delta A_{h}$ from (3.45) and $\delta \Lambda=\left(W_{f}-W_{i}\right) / M_{p}^{2}$ into (4.14) gives

$$
\delta M=\frac{r_{c} r_{h}}{r_{c}^{2}+r_{h}^{2}} M \frac{|\delta \Lambda|}{\Lambda}
$$

Analogous to the check we did on the change in $\Lambda$ (3.47), one can vary $M$ directly from (3.14), substitute in our results for the changes in $r_{h}, r_{c}$ and $\Lambda$, and find the same answer as in (4.15).

Lastly, it is interesting to assemble the terms on the right hand side of (4.14) as follows. We have that

$$
\begin{aligned}
T_{d y n} \delta S & =\frac{1}{4 G}\left[B^{-1 / 2}-\frac{W(\phi)}{M_{p}^{2}} \sqrt{B}-\frac{v \kappa_{h} \delta B_{, v}}{B}\right] \delta B \\
& =\frac{1}{4 G}\left[B^{-1 / 2}-\frac{W(\phi)}{M_{p}^{2}} \sqrt{B}\right] \delta B+\mathcal{O}(\delta B)^{2}
\end{aligned}
$$

Meanwhile,

$$
V_{h} \delta P=\frac{4 \pi B^{3 / 2}}{3} \delta(-W)
$$

so

$$
\begin{aligned}
T_{d y n} \delta S_{h}+V_{h} \delta P & =\frac{\delta B}{4 G \sqrt{B}}-2 \pi W \sqrt{B} \delta B-\frac{4 \pi}{3} B^{3 / 2} \delta W \\
& =\left.\frac{1}{2 G} \delta\left[\sqrt{B}-\frac{W}{3 M_{p}^{2}} B^{3 / 2}\right]\right|_{u=0}
\end{aligned}
$$

This quantity is defined on the black hole horizon, and if our assumptions are correct, is equal to $\delta M$ in (4.15). It also suggests that the mass is given by

$$
M=\left.\frac{1}{2 G}\left[\sqrt{B}-\frac{W}{3 M_{p}^{2}} B^{3 / 2}\right]\right|_{u=0}
$$

In the static case, where $B=r^{2}$, this is precisely the definition of $M$. Supporting this interpretation is that integrating (3.5), gives that to leading order the additional piece in the dynamical temperature is

$$
\delta B_{, v}=-\frac{1}{M_{p}^{2}} \int B \phi_{, v}^{2}=-\frac{1}{M_{p}^{2}} \int T_{v v}
$$

that is, the 'mass' contribution due to scalar accretion onto the black hole. In general, to complete the argument, we would want to show that the quantity evaluated at $u=0$ is equal to a quantity defined on future spacelike infinity.

\section{Illustrative example}

In this paper, we have derived general results for the accreting black hole. It is helpful to illustrate these with a test-case example, using our standard double well potential (2.21), 
which will allow us to explore the effects of varying the black hole mass and slow roll parameters. Recall that the solution to the slow-roll equation, $3 \gamma \phi^{\prime}=-W^{\prime}(\phi)$, for $(2.21)$ is

$$
\phi^{2}=\eta^{2} \frac{e^{H_{i}^{2} \Gamma x / 2 \gamma}}{e^{H_{i}^{2} \Gamma x / 2 \gamma}+1}
$$

We start by focussing on the black hole event horizon where $\kappa_{h} x \simeq \log \left(2 \kappa_{h} v\right)$, and compute the dynamical horizon area and temperature as a function of $\hat{v}=2 \kappa_{h} v$. Writing $a=\Gamma H_{i}^{2} / 2 \gamma \kappa_{h}$, we have

$$
\delta W=W[\phi]-W[0]=-\frac{3 H_{i}^{2} \Gamma \eta^{2}}{16} \frac{\hat{v}^{a}\left(2+\hat{v}^{a}\right)}{\left(\hat{v}^{a}+1\right)^{2}}
$$

and hence (3.42) gives

$$
\mathcal{A}=4 \pi B=\mathcal{A}_{0}\left(1-\frac{a \Delta \hat{v}}{8} \mathcal{I}[\hat{v}, a]\right)
$$

Here $\Delta=\eta^{2} / M_{p}^{2}$ represents the strength of the gravitational interaction of the scalar field, and

$$
\begin{aligned}
\mathcal{I}[\hat{v}, a] & =-\int_{\hat{v}}^{\infty} \frac{y^{a}\left(2+y^{a}\right) d y}{y^{2}\left(1+y^{a}\right)^{2}} \\
& =-\frac{\left(1+a\left(1+\hat{v}^{a}\right)\right)}{a \hat{v}\left(1+\hat{v}^{a}\right)}+\frac{1+a}{a \hat{v}}\left(1-{ }_{2} F_{1}\left[1, \frac{1}{a} ; \frac{1+a}{a} ;-\hat{v}^{-a}\right]\right)
\end{aligned}
$$

is a dimensionless integral. Meanwhile, the dynamical temperature is found to be

$$
T_{\text {dyn }}=T_{\text {init }}+\frac{a \Delta}{32 \pi}\left[\kappa_{h}\left(3 \gamma r_{h}+1\right) \frac{\hat{v}^{a}\left(2+\hat{v}^{a}\right)}{\left(1+\hat{v}^{a}\right)^{2}}+\frac{\hat{v}}{r_{h}} \mathcal{I}[\hat{v}, a]\right]
$$

Having extracted the dimensionful dependence, we can now see how the various parameters impact the evolution of the scalar and hence the black hole horizon. First, it is clear that the overall gravitational strength of the scalar, $\Delta$, simply scales the overall magnitude of the variation of the area and temperature. The parameter $a$ on the other hand not only scales the magnitude of these variations, but also their rapidity, as would be expected since $a$ is directly dependent on the rate of slow-roll of the scalar field. Given the expressions for $\gamma$ and $\kappa_{h}$, we see that

$$
a \sim \frac{\Gamma H_{i}^{2}}{2 \gamma \kappa_{h}}=\frac{\Gamma\left(r_{c}^{2} / r_{h}^{2}+r_{c} / r_{h}+1\right)}{\left(r_{c}^{2} / r_{h}^{2}+1\right)\left(r_{c} / r_{h}+2\right)}<\frac{\Gamma}{2}
$$

It is perhaps most useful to display the variations of the black hole variables as a function of the local (normalised) Eddington-Finkelstein advanced time co-ordinate on the event horizon, $\hat{V}_{E F}=H\left(t+r^{\star}\right)=\frac{H}{\kappa_{h}} \log \left[2 \kappa_{h} v\right] \sim H x$. Figure 2 shows a plot of the variation of horizon area with advanced time, while figure 3 shows the variation of temperature. In both cases, rather large values of $\Delta$ and $\Gamma$ have been chosen to emphasize the effects. The differing gradations of "slow-roll" on display arise because of the differing black hole: cosmological horizon ratios - recall that the true slow-roll parameter $\gamma$, given in (3.29), for the scalar in the presence of the black hole has a strong dependence on the geometry. 


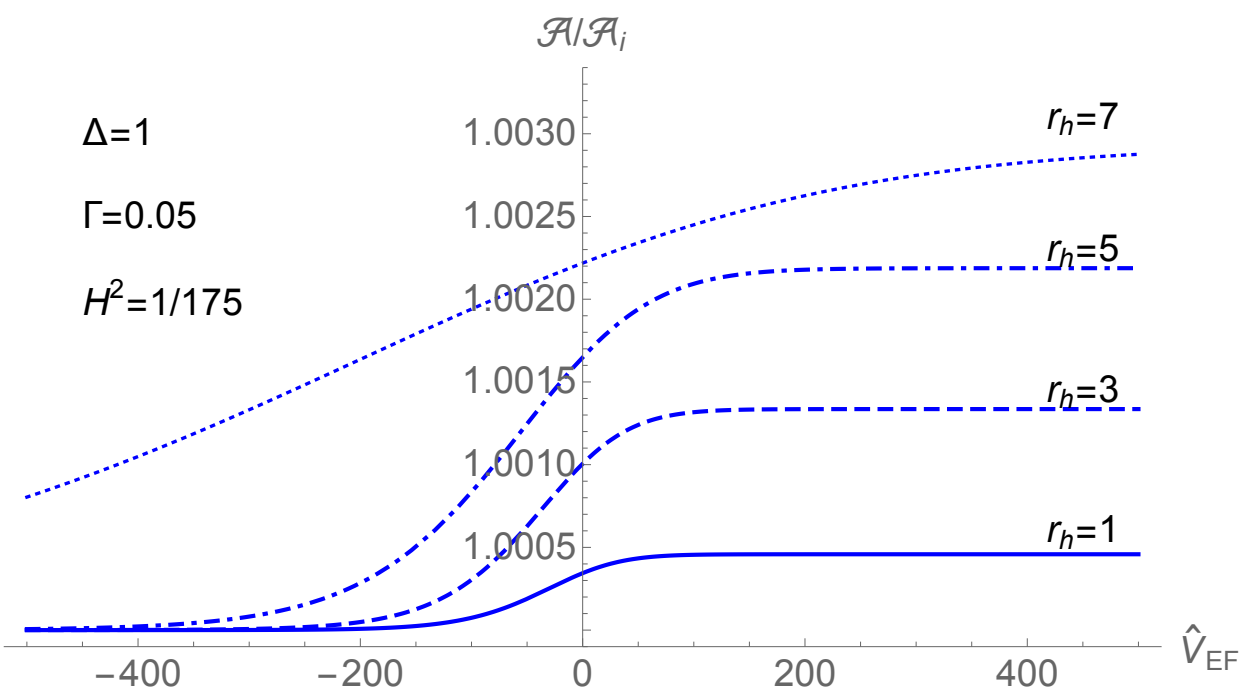

Figure 2. Illustration of evolution of the horizon area $\left(\mathcal{A}_{h} / \mathcal{A}_{i}\right)$ as a function of the EddingtonFinkelstein advanced time coordinate, scaled by the Hubble parameter, on the black hole horizon. We see that for a larger initial black hole, the increase in black hole horizon area is both larger and more gradual, than for small black holes.

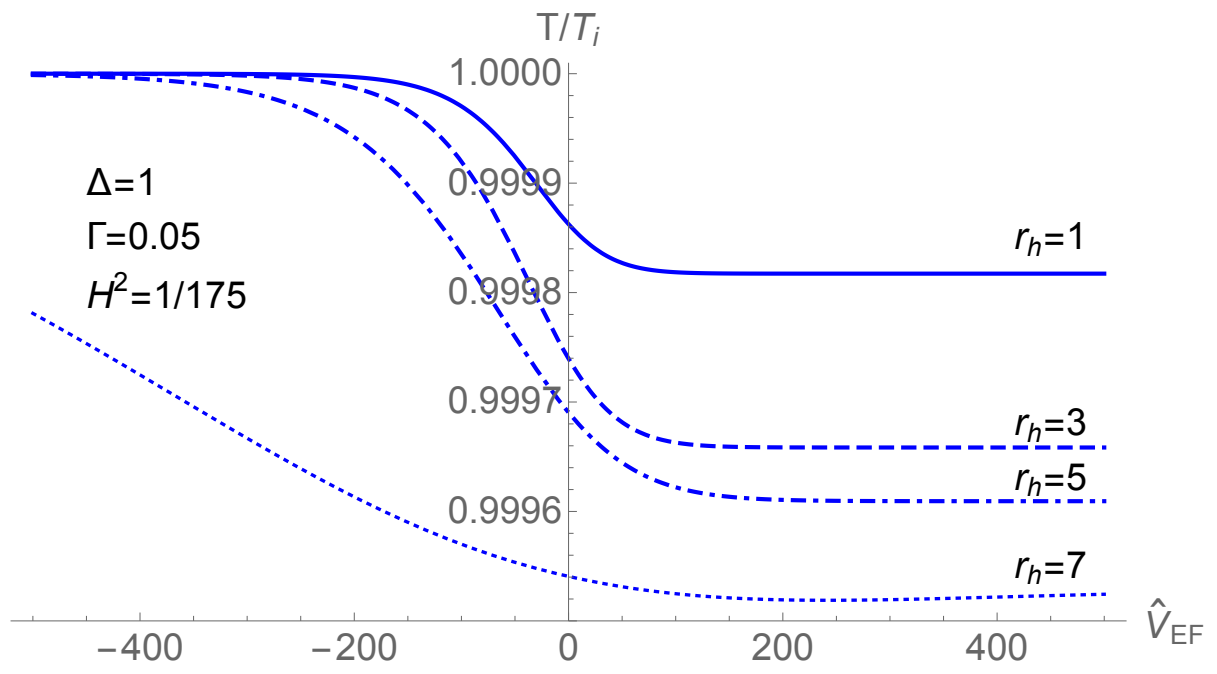

Figure 3. Illustration of evolution of temperature $\left(T / T_{i}\right)$ as a function of the Eddington-Finkelstein advanced time coordinate on the black hole horizon. We see that for larger black holes the decrease in black hole horizon temperature is both larger and more gradual than for small black holes. 


\section{Concluding remarks}

Central concepts in gravitational thermodynamics are horizon areas and temperatures, and the relations between them. The analysis presented here allows the study of these quantities in a "mildly" dynamical setting, namely the evolution from one SdS spacetime to a second SdS with a smaller cosmological constant, in a perturbative and slow-roll approximation. An advantage of these boundary conditions is that the initial and final states are equilibria with approximate Killing horizons and associated temperatures. Within these approximations we have solved the Einstein plus scalar field system to extract the growth of the black hole and cosmological horizons for a general scalar potential that has a maximum and a minimum. The results are expressed in terms of the change in the effective cosmological constant, as dictated by the potential, and geometrical properties of the initial spacetime. Using a proposed definition of dynamical temperature, the temperatures of each horizon are found to decrease between the initial and final values as the horizons grow.

One of the interesting features of the solutions is that the first law of thermodynamics (4.5), formulated between the two horizons, holds between the initial and final SdS states. This brings up two questions for further study. One is to derive the first law including the stress-energy of a scalar field, which generates additional contributions at the black hole and cosmological horizons, and verify that these contributions vanish for our solutions. Second, this result suggests that the first law might be satisfied not only between the early and late time SdS metrics, but continuously along the evolution. More strongly, is there a solution for the metric functions which illustrates that the metric is quasi-SdS at each time? In general, it would be advantageous to have explicit expressions for the full metric across the range of $r$ and $x$. This would facilitate analyzing the flow of energy-momentum throughout the volume, to test the definition of dynamical temperature by studying the near-horizon metric, and to follow the evolution of the mass-like quantity that interpolates between the initial and final mass parameters. Another interesting direction for further work is to transform the analysis to cosmological coordinates, which are likely more convenient for ascertaining how a black hole affects the important predictions of inflation, such as the spectrum of quantum perturbations and reheating.

\section{Acknowledgments}

The authors would like to thank Kostas Skenderis for helpful conversations. RG is supported in part by STFC (Consolidated Grant ST/J000407/1). RG also acknowledges support from the Wolfson Foundation and Royal Society, and Perimeter Institute for Theoretical Physics. Research at Perimeter Institute is supported by the Government of Canada through the Department of Innovation, Science and Economic Development Canada and by the Province of Ontario through the Ministry of Research, Innovation and Science.

\section{A Cosmological tension}

The ADM cosmological tension charges for an asymptotically future de Sitter spacetime were constructed in [13]. Generally, an ADM charge corresponding to an asymptotic sym- 
metry of a spacetime is defined via Hamiltonian perturbation theory [64]. We follow the general prescription presented in [41], which we briefly outline here. The construction starts with foliating the spacetime by $(D-1)$-dimensional hypersurfaces $\Sigma$ with unit normal $n^{a}$ such that the metric can be decomposed as

$$
g_{a b}=(n \cdot n) n_{a} n_{b}+s_{a b}
$$

where $n_{a} n^{a}= \pm 1$ and $s_{a b}$ denotes the induced metric on the slice(s), satisfying the orthogonality relation $s_{a b} n^{b}=0$. Let $\left(s_{a b}, \pi^{a b}\right)$ denote the Hamiltonian initial data on a slice $\Sigma$ and $\left(h_{a b}, p^{a b}\right)$ be perturbations linearized about the background denoted by $\left(\bar{s}_{a b}, \bar{\pi}^{a b}\right)$. Furthermore, let $\xi^{a}$ be a Killing vector of the background, which we project along the slice $\Sigma$ and its normal according to $\xi^{a}=F n^{a}+\beta^{a}$ such that $n_{a} \beta^{a}=0$.

The ADM charge corresponding to the Killing vector $\xi^{a}$ is then defined by an integral over a $(D-2)$-dimensional boundary at infinity on $\Sigma$ given by

$$
Q(\xi)=-\frac{1}{16 \pi G} \int_{\partial \Sigma_{\infty}} d a_{c} B^{c}
$$

where

$$
\begin{aligned}
B^{a}= & F\left(\bar{D}^{a} h-\bar{D}_{b} h^{a b}\right)-h \bar{D}^{a} F+h^{a b} \bar{D}_{b} F \\
& +\frac{1}{\sqrt{s}} \beta^{b}\left(\bar{\pi}^{c d} h_{c d} \bar{s}_{b}{ }_{b}-2 \bar{\pi}^{a c} h_{b c}-2 p^{a}{ }_{b}\right)
\end{aligned}
$$

and $\bar{D}_{a}$ is the covariant derivative operator compatible with the background metric $\bar{s}_{a b}$. Note that to simply define the charge $Q$ one only needs the symmetry (and the foliation) asymptotically. However, if the symmetry holds througout the spacetime, this set-up can then be used to prove a first law [41].

The ADM mass results when a spacetime has an asymptotic static Killing field at spatial infinity and choosing $\Sigma$ to be a timelike slice with unit timelike normal, $n_{a} n^{a}=-1$. On the other hand, the construction can be used for a cosmological spacetime with an asymptotic spatial translation Killing field $\xi$ and taking $\Sigma$ to have a unit spacelike normal, $n_{a} n^{a}=1$. Then the boundary $\partial \Sigma$ is in the asymptotic future. The resulting ADM charge is a cosmological tension.

If the spacetime is anisotropic but homogeneous, as was considered in [13], then the tensions are distinct. The inflationary spacetimes studied in this paper are isotropic and homogeneous, so there are three equal tensions. Using the formulae derived in [13] to process the boundary term (A.2) for the underdamped asymptotically de Sitter case, and averaging over a period of oscillation, gives the cosmological tension (2.37). In the overdamped and critically damped cases, the metric functions decay too slowly to balance the growth of the volume element, and the tension diverges.

Open Access. This article is distributed under the terms of the Creative Commons Attribution License (CC-BY 4.0), which permits any use, distribution and reproduction in any medium, provided the original author(s) and source are credited. 


\section{References}

[1] B.J. Carr, K. Kohri, Y. Sendouda and J. Yokoyama, New cosmological constraints on primordial black holes, Phys. Rev. D 81 (2010) 104019 [arXiv:0912.5297] [INSPIRE].

[2] B. Carr, F. Kuhnel and M. Sandstad, Primordial black holes as dark matter, Phys. Rev. D 94 (2016) 083504 [arXiv: 1607.06077] [INSPIRE].

[3] Z. Sherkatghanad and R.H. Brandenberger, The effect of primordial non-gaussianities on the seeds of super-massive black holes, arXiv:1508.00968 [INSPIRE].

[4] S. Bird et al., Did LIGO detect dark matter?, Phys. Rev. Lett. 116 (2016) 201301 [arXiv: 1603.00464] [INSPIRE].

[5] M. Kawasaki, A. Kusenko, Y. Tada and T.T. Yanagida, Primordial black holes as dark matter in supergravity inflation models, Phys. Rev. D 94 (2016) 083523 [arXiv:1606.07631] [INSPIRE].

[6] R. Bean and J. Magueijo, Could supermassive black holes be quintessential primordial black holes?, Phys. Rev. D 66 (2002) 063505 [astro-ph/0204486] [INSPIRE].

[7] K. Kannike, L. Marzola, M. Raidal and H. Veermäe, Single field double inflation and primordial black holes, JCAP 09 (2017) 020 [arXiv: 1705.06225] [INSPIRE].

[8] V. Domcke, F. Muia, M. Pieroni and L.T. Witkowski, PBH dark matter from axion inflation, JCAP 07 (2017) 048 [arXiv: 1704.03464] [INSPIRE].

[9] J. Georg and S. Watson, A preferred mass range for primordial black hole formation and black holes as dark matter revisited, arXiv:1703.04825 [INSPIRE].

[10] E. Mediavilla, J. Jiménez-Vicente, J.A. Muñoz, H. Vives-Arias and J. Calderón-Infante, Limits on the mass and abundance of primordial black holes from quasar gravitational microlensing, Astrophys. J. 836 (2017) L18 [arXiv: 1702.00947] [INSPIRE].

[11] D. Kastor, S. Ray and J. Traschen, Enthalpy and the mechanics of AdS black holes, Class. Quant. Grav. 26 (2009) 195011 [arXiv:0904.2765] [InSPIRE].

[12] B.P. Dolan, D. Kastor, D. Kubiznak, R.B. Mann and J. Traschen, Thermodynamic volumes and isoperimetric inequalities for de Sitter black holes, Phys. Rev. D 87 (2013) 104017 [arXiv: 1301.5926] [INSPIRE].

[13] D. Kastor, S. Ray and J. Traschen, Genuine cosmic hair, Class. Quant. Grav. 34 (2017) 045003 [arXiv: 1608.04641] [INSPIRE].

[14] D. Sudarsky and J.A. Gonzalez, On black hole scalar hair in asymptotically anti-de Sitter space-times, Phys. Rev. D 67 (2003) 024038 [gr-qc/0207069] [INSPIRE].

[15] M. Henneaux, C. Martinez, R. Troncoso and J. Zanelli, Black holes and asymptotics of $2+1$ gravity coupled to a scalar field, Phys. Rev. D 65 (2002) 104007 [hep-th/0201170] [INSPIRE].

[16] T. Hertog and K. Maeda, Black holes with scalar hair and asymptotics in $N=8$ supergravity, JHEP 07 (2004) 051 [hep-th/0404261] [INSPIRE].

[17] C. Martinez, R. Troncoso and J. Zanelli, Exact black hole solution with a minimally coupled scalar field, Phys. Rev. D 70 (2004) 084035 [hep-th/0406111] [INSPIRE].

[18] E. Radu and D.H. Tchrakian, New hairy black hole solutions with a dilaton potential, Class. Quant. Grav. 22 (2005) 879 [hep-th/0410154] [INSPIRE]. 
[19] E. Winstanley, Dressing a black hole with non-minimally coupled scalar field hair, Class. Quant. Grav. 22 (2005) 2233 [gr-qc/0501096] [INSPIRE].

[20] F. Faedo, D. Klemm and M. Nozawa, Hairy black holes in $N=2$ gauged supergravity, JHEP 11 (2015) 045 [arXiv: 1505.02986] [INSPIRE].

[21] A. Anabalon, D. Astefanesei, D. Choque and C. Martinez, Trace anomaly and counterterms in designer gravity, JHEP 03 (2016) 117 [arXiv:1511.08759] [INSPIRE].

[22] T. Hertog and G.T. Horowitz, Designer gravity and field theory effective potentials, Phys. Rev. Lett. 94 (2005) 221301 [hep-th/0412169] [INSPIRE].

[23] M. Henneaux, C. Martinez, R. Troncoso and J. Zanelli, Asymptotic behavior and Hamiltonian analysis of anti-de Sitter gravity coupled to scalar fields, Annals Phys. $\mathbf{3 2 2}$ (2007) 824 [hep-th/0603185] [INSPIRE].

[24] J. Kaplan and J. Wang, An effective theory for holographic RG flows, JHEP 02 (2015) 056 [arXiv: 1406.4152] [INSPIRE].

[25] K. Skenderis and P.K. Townsend, Hidden supersymmetry of domain walls and cosmologies, Phys. Rev. Lett. 96 (2006) 191301 [hep-th/0602260] [InSPIRE].

[26] D. Bazeia, F.A. Brito and F.G. Costa, First-order framework and domain-wall/brane-cosmology correspondence, Phys. Lett. B 661 (2008) 179 [arXiv:0707.0680] [INSPIRE].

[27] P. McFadden and K. Skenderis, The holographic universe, J. Phys. Conf. Ser. 222 (2010) 012007 [arXiv: 1001.2007] [INSPIRE].

[28] A. Bzowski, P. McFadden and K. Skenderis, Holography for inflation using conformal perturbation theory, JHEP 04 (2013) 047 [arXiv: 1211.4550] [INSPIRE].

[29] U. Kol, On the dual flow of slow-roll inflation, JHEP 01 (2014) 017 [arXiv:1309.7344] [INSPIRE].

[30] S. Chadburn and R. Gregory, Time dependent black holes and scalar hair, Class. Quant. Grav. 31 (2014) 195006 [arXiv: 1304.6287] [INSPIRE].

[31] S.A. Hayward, R. Di Criscienzo, L. Vanzo, M. Nadalini and S. Zerbini, Local Hawking temperature for dynamical black holes, Class. Quant. Grav. 26 (2009) 062001 [arXiv: 0806.0014] [INSPIRE].

[32] L. Girardello, M. Petrini, M. Porrati and A. Zaffaroni, Novel local CFT and exact results on perturbations of $N=4$ super Yang-Mills from AdS dynamics, JHEP 12 (1998) 022 [hep-th/9810126] [INSPIRE].

[33] D.Z. Freedman, S.S. Gubser, K. Pilch and N.P. Warner, Renormalization group flows from holography supersymmetry and a $c$ theorem, Adv. Theor. Math. Phys. 3 (1999) 363 [hep-th/9904017] [INSPIRE].

[34] K. Skenderis and P.K. Townsend, Gravitational stability and renormalization group flow, Phys. Lett. B 468 (1999) 46 [hep-th/9909070] [INSPIRE].

[35] K. Skenderis and P.K. Townsend, Hamilton-Jacobi method for curved domain walls and cosmologies, Phys. Rev. D 74 (2006) 125008 [hep-th/0609056] [INSPIRE].

[36] A. Kehagias and K. Tamvakis, Localized gravitons, gauge bosons and chiral fermions in smooth spaces generated by a bounce, Phys. Lett. B 504 (2001) 38 [hep-th/0010112] [INSPIRE]. 
[37] K. Skenderis and P.K. Townsend, Pseudo-supersymmetry and the domain-wall/cosmology correspondence, J. Phys. A 40 (2007) 6733 [hep-th/0610253] [INSPIRE].

[38] A.V. Frolov and L. Kofman, Inflation and de Sitter thermodynamics, JCAP 05 (2003) 009 [hep-th/0212327] [INSPIRE].

[39] A.R. Liddle, P. Parsons and J.D. Barrow, Formalizing the slow roll approximation in inflation, Phys. Rev. D 50 (1994) 7222 [astro-ph/9408015] [INSPIRE].

[40] R.M. Wald, Asymptotic behavior of homogeneous cosmological models in the presence of a positive cosmological constant, Phys. Rev. D 28 (1983) 2118 [inSPIRE].

[41] J.H. Traschen and D. Fox, Tension perturbations of black brane space-times, Class. Quant. Grav. 21 (2004) 289 [gr-qc/0103106] [InSPIRE].

[42] B. Mahmoud El-Menoufi, B. Ett, D. Kastor and J. Traschen, Gravitational tension and thermodynamics of planar AdS spacetimes, Class. Quant. Grav. 30 (2013) 155003 [arXiv: 1302.6980] [INSPIRE].

[43] P. Breitenlohner and D.Z. Freedman, Stability in gauged extended supergravity, Annals Phys. 144 (1982) 249 [INSPIRE].

[44] G.C. McVittie, The mass-particle in an expanding universe, Mon. Not. Roy. Astron. Soc. 93 (1933) 325 [INSPIRE].

[45] D. Kastor and J.H. Traschen, Cosmological multi-black hole solutions, Phys. Rev. D 47 (1993) 5370 [hep-th/9212035] [INSPIRE].

[46] G.W. Gibbons and K.-I. Maeda, Black holes in an expanding universe, Phys. Rev. Lett. 104 (2010) 131101 [arXiv:0912.2809] [INSPIRE].

[47] T. Jacobson, Primordial black hole evolution in tensor scalar cosmology, Phys. Rev. Lett. 83 (1999) 2699 [astro-ph/9905303] [INSPIRE].

[48] H. Saida and J. Soda, Black holes and a scalar field in expanding universe, Class. Quant. Grav. 17 (2000) 4967 [gr-qc/0006058] [INSPIRE].

[49] T. Harada and B.J. Carr, Growth of primordial black holes in a universe containing a massless scalar field, Phys. Rev. D 71 (2005) 104010 [astro-ph/0412135] [INSPIRE].

[50] J. Sultana and C.C. Dyer, Cosmological black holes: a black hole in the Einstein-de Sitter universe, Gen. Rel. Grav. 37 (2005) 1347 [INSPIRE].

[51] V. Faraoni and A. Jacques, Cosmological expansion and local physics, Phys. Rev. D 76 (2007) 063510 [arXiv:0707.1350] [INSPIRE].

[52] M. Carrera and D. Giulini, On the influence of global cosmological expansion on the dynamics and kinematics of local systems, arXiv:0810.2712 [INSPIRE].

[53] M.G. Rodrigues and A. Saa, Accretion of nonminimally coupled scalar fields into black holes, Phys. Rev. D 80 (2009) 104018 [arXiv:0909.3033] [INSPIRE].

[54] B.J. Carr, T. Harada and H. Maeda, Can a primordial black hole or wormhole grow as fast as the universe?, Class. Quant. Grav. 27 (2010) 183101 [arXiv:1003.3324] [INSPIRE].

[55] L.A. Urena-Lopez and L.M. Fernandez, Black holes and the absorption rate of cosmological scalar fields, Phys. Rev. D 84 (2011) 044052 [arXiv:1107.3173] [INSPIRE]. 
[56] D.C. Guariento, M. Fontanini, A.M. da Silva and E. Abdalla, Realistic fluids as source for dynamically accreting black holes in a cosmological background, Phys. Rev. D 86 (2012) 124020 [arXiv: 1207.1086] [INSPIRE].

[57] M.G. Rodrigues and A.E. Bernardini, Accretion of non-minimally coupled generalized Chaplygin gas into black holes, Int. J. Mod. Phys. D 21 (2012) 1250075 [arXiv:1208.1572] [INSPIRE].

[58] E. Abdalla, N. Afshordi, M. Fontanini, D.C. Guariento and E. Papantonopoulos, Cosmological black holes from self-gravitating fields, Phys. Rev. D 89 (2014) 104018 [arXiv: 1312.3682] [INSPIRE].

[59] E.O. Babichev, V.I. Dokuchaev and Y.N. Eroshenko, Black holes in the presence of dark energy, Phys. Usp. 56 (2013) 1155 [Usp. Fiz. Nauk 189 (2013) 1257] [arXiv:1406.0841] [INSPIRE].

[60] N. Afshordi, M. Fontanini and D.C. Guariento, Horndeski meets McVittie: a scalar field theory for accretion onto cosmological black holes, Phys. Rev. D 90 (2014) 084012 [arXiv: 1408.5538] [INSPIRE].

[61] A.-C. Davis, R. Gregory and R. Jha, Black hole accretion discs and screened scalar hair, JCAP 10 (2016) 024 [arXiv: 1607.08607] [INSPIRE].

[62] A.V. Frolov, J.T. Gálvez Ghersi and A. Zucca, Unscreening scalarons with a black hole, Phys. Rev. D 95 (2017) 104041 [arXiv: 1704.04114] [INSPIRE].

[63] P. Bowcock, C. Charmousis and R. Gregory, General brane cosmologies and their global space-time structure, Class. Quant. Grav. 17 (2000) 4745 [hep-th/0007177] [INSPIRE].

[64] T. Regge and C. Teitelboim, Role of surface integrals in the Hamiltonian formulation of general relativity, Annals Phys. 88 (1974) 286 [INSPIRE]. 